\title{
Freshwater fishes of the Bahia State, Northeastern Brazil
}

\author{
André Teixeira Silva ${ }^{1 *}{ }^{\oplus}$, Ricardo Jucá Chagas ${ }^{1}{ }^{\circledR}$, Alexandre Clistenes de Alcântara Santos ${ }^{2}{ }^{\circledR}$, Angela

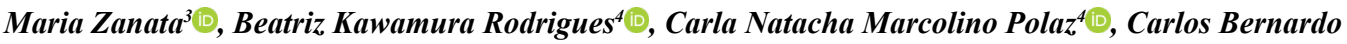

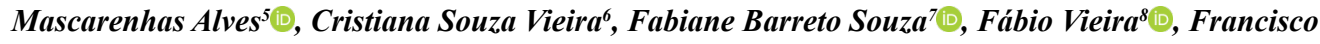 \\ Alexandre Costa Sampaio ${ }^{1}$, Harildon Ferreira ${ }^{9}$, Hilda Susele Rodrigues Alves ${ }^{9}$, Luisa Maria \\ Sarmento-Soares $^{10} \mathbb{D}^{\mathbb{D}}$, Marianna Pinho ${ }^{10}$, Ronaldo Fernando Martins-Pinheiro ${ }^{11} \mathbb{D}$, Sergio Maia Queiroz Lima ${ }^{12} \mathbb{D}^{\mathbb{D}}$, \\ Sofia Campiolo ${ }^{9}$ \& Priscila Camelier ${ }^{3}[\mathbb{D}$ \\ ${ }^{1}$ Universidade Estadual do Sudoeste da Bahia, Departamento de Ciências Biológicas, Av. José Moreira \\ Sobrinho, s/n, Jequiezinho, 45205-490, Jequié, BA, Brasil. \\ ${ }^{2}$ Universidade Estadual de Feira de Santana, Departamento de Ciências Biológicas, Av. Universitária s/n, Novo \\ Horizonte, 44036-900, Feira de Santana, BA, Brasil. \\ ${ }^{3}$ Universidade Federal da Bahia, Instituto de Biologia, Programa de Pós-Graduação em Biodiversidade e \\ Evolução, Rua Barão de Geremoabo, 147, Ondina, 40170-290, Salvador, BA, Brasil. \\ ${ }^{4}$ Instituto Chico Mendes de Conservação da Biodiversidade, Centro Nacional de Pesquisa e Conservação da \\ Biodiversidade Aquática Continental, Rodovia Prefeito Euberto Nemésio Pereira de Godoy (SP 201), km 6,5, \\ Cachoeira de Emas, 13630-970, Pirassununga, SP, Brasil. \\ ${ }^{5}$ Universidade Federal de Minas Gerais, Av. Antônio Carlos, 6627, Pampulha, 31270-901, Belo Horizonte, MG, Brasil. \\ ${ }^{6}$ Secretaria de Ciência Tecnologia e Inovação do Estado da Bahia, $5^{a}$ Avenida, Plataforma II, Ala B - $1^{\circ}$ andar, \\ CAB, 41745-004, Salvador, BA, Brasil. \\ ${ }^{7}$ Instituto Federal de Educação, Ciência e Tecnologia Baiano, Campus Santa Inês, BR 420, Rodovia Santa Inês, Ubaíra, \\ Zona Rural, 45320-000, Santa Inês, BA, Brasil. \\ ${ }^{8}$ Universidade Federal de Minas Gerais, Instituto de Ciências Biológicas, Departamento de Zoologia, Av. Pres. \\ Antônio Carlos, 6627, Pampulha, 31270-901, Belo Horizonte, MG, Brasil. \\ ${ }^{9}$ Universidade Estadual de Santa Cruz, Departamento de Ciências Biológicas, Rod. Ilhéus-Itabuna Km16, \\ Salobrinho, 45660-000, Ilhéus, BA, Brasil. \\ ${ }^{10}$ Universidade Federal do Espirito Santo (UFES), Programa de Pós-Graduação em Biologia Animal \\ (PPGBAN), Campus de Goiabeiras, 29043-900, Vitória, ES, Brasil. \\ ${ }^{11}$ Instituto Nacional da Mata Atlântica, Laboratório de Zoologia, Av. José Ruschi, 4, Centro, 29650-000, Santa Teresa, \\ ES, Brasil. \\ ${ }^{12}$ Universidade Federal do Rio Grande do Norte, Departamento de Botânica e Zoologia, Av. Senador Salgado \\ Filho 3000, Lagoa Nova, 56078-970, Natal, RN, Brasil. \\ *Corresponding author: André Teixeira Silva,e-mail: silva.at@gmail.com
}

SILVA, A.T., CHAGAS, R.J., SANTOS, A.C.A., ZANATA, A.M., RODRIGUES, B.K., POLAZ, C.N.M., ALVES, C.B.M., VIEIRA, C.S., SOUZA, F.B., VIEIRA, F., SAMPAIO, F.A.C., FERREIRA, H., ALVES, H.R.A., SARMENTO-SOARES, L.M., PINHO, M., MARTINS-PINHEIRO, R.F., LIMA, S.M.Q., CAMPIOLO, S., CAMELIER, P. Freshwater fishes of the Bahia State, Northeastern Brazil. Biota Neotropica 20(4): e20200969. https://doi.org/10.1590/1676-0611-BN-2020-0969

\begin{abstract}
This work was carried out from the assessment of the conservation status of the freshwater ichthyofauna from Bahia State. The inventory data and species distribution were obtained from the specialized scientific literature and representative ichthyological collections. A total of 281 native species was recorded in Bahia State, distributed in the Northeastern Mata Atlantica (NMA) and São Francisco (SFR) freshwater ecoregions. There was a larger number of species in the NMA (187 spp.), composed by several coastal basins, than in the SFR (134 spp.), composed by São Francisco river basin. Among the 30 families recorded, Characidae and Rivulidae were the most representative, with 53 and 48 species, respectively. The conservation status of 214 species was assessed and 33 of them (15\%) were included in the IUCN threat categories. Of these, 11 species were classified as vulnerable (VU), 12 as endangered $(\mathrm{EN})$, and 10 as critically endangered $(\mathrm{CR})$. Most threatened species $(\mathrm{n}=14)$ belongs to the family Rivulidae. The larger number of threatened species in the NMA: $(n=23)$ is mainly related to the high endemism of restricted-range species associated with the human occupation impacts along the coastal regions. In the SFR, most of threatened species are annual killifishes, which are locally disappearing due to increasing degradation of their temporary habitats. Keywords: biodiversity, threatened species, endemism, Northeastern Mata Atlantica ecoregion, São Francisco river basin.
\end{abstract}




\section{Peixes de água doce do Estado da Bahia, Nordeste do Brasil}

Resumo: Este trabalho foi realizado a partir da avaliação do estado de conservação da ictiofauna de água doce do Estado da Bahia. Os dados de inventário e distribuição das espécies foram obtidos a partir da literatura científica especializada e de coleções ictiológicas representativas. Um total de 281 espécies nativas foi registrado no Estado da Bahia, distribuídas nas ecorregiões de água doce Mata Atlântica Nordeste (NMA) e São Francisco (SFR). A riqueza de espécies foi maior na NMA (187 spp.), composta por diversas bacias costeiras, do que na SFR (134 spp.), composta pela bacia do rio São Francisco. Das 30 famílias registradas, as mais representativas foram Characidae e Rivulidae, com 53 e 48 espécies, respectivamente. O estado de conservação de 214 espécies foi avaliado e 33 (15\%) destas foram classificadas em alguma categoria de ameaça da IUCN. Destas, 11 foram classificadas como vulneráveis (VU), 12 em perigo (EN) e 10 criticamente em perigo (CR). A maioria das espécies ameaçadas ( $\mathrm{n}=$ 14) pertence à família Rivulidae. O elevado número de espécies ameaçadas na NMA: $(\mathrm{n}=23)$ está associado ao alto endemismo das espécies com distribuição restrita e aos impactos da ocupação humana ao longo da região costeira. Na SFR, a maioria das espécies ameaçadas é de peixes anuais, que estão localmente desaparecendo devido à degradação crescente de seus habitats temporários.

Palavras-chave: biodiversidade, espécies ameaçadas, endemismo, Ecorregião Mata Atlântica Nordeste, bacia do rio São Francisco.

\section{Introduction}

The Neotropical region harbors the most diverse freshwater fish fauna in the world, with more than 5,700 described species, although the final number may exceed 8,000 species (Albert et al. 2011, Reis 2013, Bertaco et al. 2016, Reis et al. 2016). Putting this number into perspective, Neotropical freshwater fishes account for about one in five of the world's fish species, or approximately $10 \%$ of all vertebrate species (Albert \& Reis 2011). According to Ribeiro (2006), the reasons for such a marked diversity are likely to be both historical and ecological, a result of millions of years of evolution from the breakup of Gondwana to the present day.

It is often claimed that freshwater ecosystems are the most endangered in the world (Sala et al. 2000, Dudgeon et al. 2006, Nogueira et al. 2010). As in other freshwater regions, South American fishes are threatened by overexploitation, flow modification, habitat destruction, species invasion, pollution, eutrophication, and siltation (Lévêque et al. 2008). Several fish conservation initiatives are underway in South America, including an ambitious program that assesses the conservation status of all Brazilian fish species (Reis 2013). This program resulted in the Brazilian list of threatened fishes and aquatic invertebrates, known as the Brazilian Red List (MMA 2014). Along with this national task, some parallel regional projects of conservation assessment of continental fishes have also been conducted (e.g., Rosa et al. 2003, Alves \& Leal 2010, Reis et al. 2016).

In 2013, one of these regional initiatives was carried out in Bahia State, northeastern Brazil, and the Instituto Chico Mendes de Conservação da Biodiversidade (ICMBio) in collaboration with the Secretaria do Meio Ambiente do Estado da Bahia (SEMA), and several ichthyologists completed a comprehensive conservation assessment of the freshwater ichthyofauna in the state. It is important to highlight that the results of this regional workshop became the basis for the publishing of the list of threatened freshwater fish species reported from Bahia state (SEMA 2017). Bahia, one of the 27 federative units of Brazil, is the fifth largest state by area, with more than $564.000 \mathrm{~km}^{2}$ and 417 municipalities (IBGE 2019).
This state partially encompasses two freshwater ecoregions as proposed by Abell et al. (2008), the São Francisco (SFR, ecoregion 327) and Northeastern Mata Atlantica (NMA, ecoregion 328) ecoregions (Figure 1). According to these authors, a freshwater ecoregion is defined as a large area encompassing one or more freshwater systems with a distinct assemblage of natural freshwater communities and species.

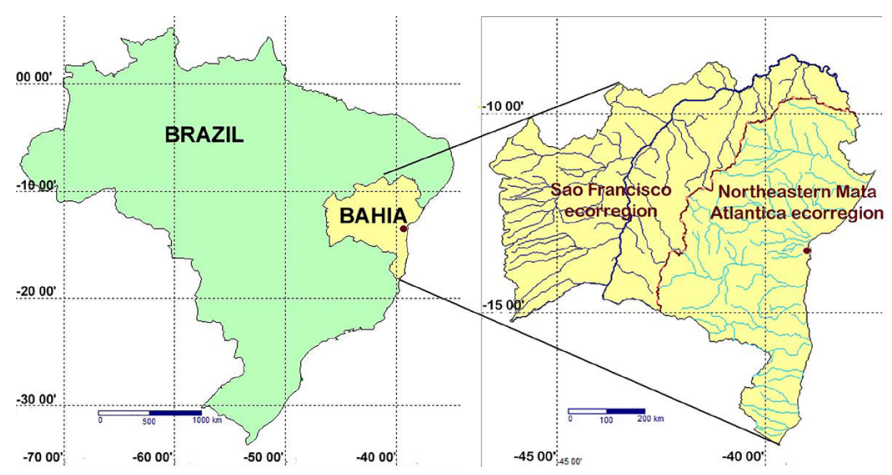

Figure 1. Localization and river drainages across Bahia state. Parts of the São Francisco (in dark blue) and Northeastern Mata Atlantica (in light blue) freshwater ecoregions in Bahia State, Northeastern Brazil, are shown. Red dotted line indicates the boundaries between Northeastern Mata Atlantica ecoregion and São Francisco ecoregion.

Although political geographic boundaries are useless for biological organisms, national or regional species lists are important for monitoring and conservation planning efforts (Silveira et al. 2010, Hortal et al. 2015). In this sense, any conservation initiative starts from the identification of the species that will be protect by such initiative. However, that apparently simple and basic information is absent or incomplete for diverse regions of Brazil. For example, the procedure of compilation of fish species that occur in rivers that drain Bahia state to evaluation of conservation status took several weeks of hard work previously to 2013 workshop, and involved the participation of several experts. 
Before that effort, the knowledge about the species that occur in Bahia rivers was dispersed in some studies which focused on description of new species (e.g., Garavello 1977, Malabarba et al. 2004, Costa 2007 , Zanata \& Serra 2010), or representing the fish species inventory of some basins or part of them (e.g., Sato \& Godinho 1999, Godinho \& Godinho 2003, Rosa et al. 2003, Santos 2005, Sarmento-Soares et al. 2007, Barbosa \& Soares 2009, Burger et al., 2011).

To make available the results of the list of threatened freshwater fishes of Bahia State elaborated during the aforementioned 2013 workshop, we report here the first checklist of freshwater fish species of the Bahia state. This list includes species from the SFR and NMA ecoregions described at present, as well the conservation status for those evaluate in 2013 Workshop. Without the pretense of being a definitive list, it is a starting point that should be broadened and updated in order to compile the scattered information available.

\section{Material and methods}

\section{Study area}

Freshwater fish species from stretches and tributaries in the SFR and NMA ecoregions of Bahia State (Figure 1) were analyzed. The SFR ecoregion is equivalent to the São Francisco basin, the third largest Neotropical hydrographic basin which is enclosed in an area of more than $630,000 \mathrm{~km}^{2}$ (Sato \& Godinho 1999, Kohler 2003, Langeani et al. 2009). The São Francisco is the longest river running entirely in Brazil, corresponding to more than $7.5 \%$ of the Brazilian territory (Godinho \& Godinho 2003, Pompeu \& Godinho 2006). With more than 2,500 $\mathrm{km}$ of extension (Godinho \& Godinho 2003, Langeani et al. 2009), the São Francisco river and its tributaries drain three biomes (Caatinga, Cerrado and, peripherally, Atlantic Forest) crossing six states (from its source to mouth: Minas Gerais, Goiás, Bahia, Pernambuco, Alagoas, and Sergipe) plus the Federal District (Paiva 1982, Godinho \& Godinho 2003, Langeani et al. 2009). From its headwaters in the Serra da Canastra, located in the central-western part of Minas Gerais State (at about 1,200-1,500 m above sea level), to its mouth, between Alagoas and Sergipe, the river drains more than 500 municipalities (Kohler 2003, Sato \& Godinho, 2003). Although not entirely located in Bahia State, the São Francisco is the largest and one of the most important rivers of the state.

The NMA, in turn, comprises all coastal drainages in eastern Brazil, from the Sergipe river in the north to the Itabapoana river in the south (Camelier \& Zanata 2014a). To the west, the NMA ecoregion is limited by the watershed border with the São Francisco river basin, along the Serra do Espinhaço up to the north, and by the Paraíba do Sul river basin in the south (Hales \& Petry 2013). This ecoregion includes more than 25 isolated basins (Camelier \& Zanata 2014a), draining the eastern slopes of the Brazilian crystalline shield directly into the Atlantic Ocean. From north to south, the main drainages of the NMA in Bahia State are: Vaza-Barris, Real, Itapicuru, Inhambupe, Pojuca, Paraguaçu, Jequiriçá, Contas, Cachoeira, Almada, Una, Pardo, Jequitinhonha, Buranhém, Frades, Jucuruçu, Itanhém, Peruípe, and Mucuri (Langeani et al. 2009, Camelier \& Zanata 2014a).
These drainages, along with several other smaller isolated basins of the NMA, are separated by the scarped, mountainous landscapes of the eastern margin of the Brazilian shield (Ribeiro 2006). The NMA is mainly inserted in the Atlantic Forest biome, although the northern drainages from the upper Contas to the Vaza-Barris river basins are partially under the influence of the semiarid Caatinga (Rosa et al. 2003, Camelier \& Zanata 2014a, Lima et al., 2017).

\section{Species inventory and list compilation}

The species inventory and information about their distributions were obtained by consulting different sources in the reliable specialized scientific literature (i.e. original descriptions, taxonomic reviews, phylogenetic studies, and species catalogues) and representative ichthyological collections such as Instituto Nacional da Mata Atlântica (formerly Museu de Biologia Professor Mello Leitão-MBML), Museu de Ciências e Tecnologia da Pontifícia Universidade Católica do Rio Grande do Sul (MCP), Museu Nacional do Rio de Janeiro (MNRJ), Museu de Zoologia da Universidade de São Paulo (MZUSP), Museu de História Natural da Bahia (UFBA), and Universidade Federal do Rio Grande do Norte (UFRN). Data were obtained from these fish collections or by searches in the national system of information on ichthyological collections (e.g., SIBIP/NEODAT III 2019, CRIA 2019) and, eventually, checked by consulting specialists. Taxonomic classification and species naming were determined mainly according to Fricke et al. (2020); for the species of Characidae, according to Mirande (2010).

To elaborate the list supporting this study, only freshwater fish species formally described until December 2019 were considered. Despite the fact that freshwater species were defined as those known to spend a significant part of their life cycle in low salinity $(<0.5 \mathrm{ppt})$ continental waters (Myers 1949; Berra 2001), in the present study only primary freshwater fishes were considered.

The International Union for Conservation of Nature (IUCN) criteria were used to asses the conservation status of the species (IUCN 2012). The conservation status of species already evaluated according to these criteria was updated to the state of Bahia, which represented the regional assessment. Since the regional workshop for the evaluation of endangered freshwater fish species in Bahia occurred in November 2013, species described after this period did not have their conservation status assessed. The only exception is Rhamdiopsis sp., an undescribed troglobitic species whose description process is well advanced (M. E. Bichuette pers. comm.). Subterranean ecosystems and their biota pose special problems for conservation due to their intrinsic fragility and the distinctive features of subterranean communities, such as a high degree of endemism and morphological, ecological and behavioral specialization (Trajano \& Bichuette 2010). Therefore, the assessment of the conservation status of Rhamdiopsis sp. has become necessary.

Species whose known distribution is restricted to the stretches of the basins draining Bahia State were classified as endemic. It should be noted that endemic species from the ecoregions analyzed (SFR or NMA), which are known to occur in other states (e.g., Sergipe, Minas Gerais, Espírito Santo), were not classified as endemic. Non-native species recorded in basins of the Bahia State were included in the list, but their conservation status was not evaluated. 


\section{Results}

\section{Ichthyofauna composition}

A total of 281 native freshwater fish species was recorded along the drainages of Bahia State (Table 1), accounting for about $9 \%$ of all species $(3,116)$ known to occur in Brazil (ICMBio 2014). Of the taxa listed in this study, one subfamily (Copionodontinae), nine genera (Copionodon, Glaphyropoma, Hirtella, Kalyptodoras, Mucurilebias, Myxiops, Ophthalmolebias, Prorivulus, and Pseudotatia), and 126 species are only known from river basins draining Bahia State. Most of the native species are from the NMA, with 187 species occurring in Bahia. In stretches of the SFR draining the state, 134 native species were listed (Table 1).

Table 1. List of freshwater fish species of Bahia State and their conservation status according to state (SEMA 2017) and national assessments (MMA 2014), IUCN criteria and ecoregion (NMA, Northeastern Mata Atlantica; SFR, São Francisco), as well as their respective indication of origin (X, native species but not endemic to the Bahia part of the ecoregion; END, endemic to the Bahia part of the ecoregion; NNA, non-native species).

\begin{tabular}{|c|c|c|c|c|c|c|c|}
\hline \multirow{2}{*}{ ORDER/Family/Species } & \multicolumn{2}{|c|}{ SEMA 2017} & \multicolumn{2}{|c|}{ ММА 2014} & \multirow{2}{*}{ SFR } & \multirow{2}{*}{ NMA } & \multirow{2}{*}{ Voucher / Source } \\
\hline & Status & IUCN Criteria & Status & IUCN Criteria & & & \\
\hline \multicolumn{8}{|l|}{ CLUPEIFORMES } \\
\hline $\begin{array}{l}\text { Anchoviella vaillanti } \\
\text { (Steindachner 1908) }\end{array}$ & $\mathrm{LC}$ & & $\mathrm{LC}$ & & $\mathrm{X}$ & & MZUSP 54604, MCP 16611 \\
\hline \multicolumn{8}{|l|}{ CHARACIFORMES } \\
\hline $\begin{array}{l}\text { Acestrorhynchus britskii } \\
\text { Menezes } 1969\end{array}$ & $\mathrm{LC}$ & & $\mathrm{LC}$ & & $\mathrm{X}$ & & MZUSP 83828, MZUSP 54683 \\
\hline $\begin{array}{l}\text { Acestrorhynchus falcatus } \\
\text { (Bloch 1794) }\end{array}$ & $\mathrm{LC}$ & & $\mathrm{LC}$ & & & $\mathrm{X}$ & $\begin{array}{l}\text { MNRJ 21754, MZUSP } 102488, \\
\text { MZUSP 102557, UFBA } 4507\end{array}$ \\
\hline $\begin{array}{l}\text { Acestrorhynchus lacustris } \\
\text { (Lütken 1875) }\end{array}$ & $\mathrm{LC}$ & & $\mathrm{LC}$ & & $\mathrm{X}$ & $\mathrm{X}$ & $\begin{array}{c}\text { MZUSP 57172, MZUSP } 28770, \text { UFBA } \\
\text { 2647, UFBA } 4946\end{array}$ \\
\hline Leporellus pictus (Kner 1850) & $\mathrm{NE}$ & & $\mathrm{NE}$ & & $\mathrm{X}$ & & UNT 13927; UNT 9561 \\
\hline $\begin{array}{l}\text { Leporellus vittatus } \\
\text { (Valenciennes 1850) }\end{array}$ & $\mathrm{LC}$ & & $\mathrm{LC}$ & & $\mathrm{X}$ & & MZUSP 54659, UFBA 833 \\
\hline $\begin{array}{l}\text { Leporinus bahiensis } \\
\text { Steindachner } 1875\end{array}$ & $\mathrm{LC}$ & & $\mathrm{LC}$ & & & END & $\begin{array}{l}\text { UFBA } 2645 \text {, UFBA } 2954, \text { UFBA } 1946, \\
\text { UFBA } 4456, \text { UFBA } 4258, \text { UFBA } 4483\end{array}$ \\
\hline $\begin{array}{l}\text { Leporinus copelandii } \\
\text { Steindachner } 1875\end{array}$ & $\mathrm{LC}$ & & $\mathrm{LC}$ & & & $\mathrm{X}$ & $\begin{array}{c}\text { MZUSP 88513, UFBA 2828, UFBA } \\
\text { 5123, UFBA } 5666\end{array}$ \\
\hline $\begin{array}{l}\text { Leporinus melanopleura } \\
\text { Günther } 1864\end{array}$ & $\mathrm{LC}$ & & $\mathrm{LC}$ & & & END & $\begin{array}{c}\text { BMNH 1863, MZUSP 111246, UFBA } \\
\text { 4282, UFBA 5103, UFBA } 8052\end{array}$ \\
\hline $\begin{array}{l}\text { Megaleporinus brinco } \\
\text { (Birindelli, Britski \& Garavello 2013) }\end{array}$ & $\mathrm{LC}$ & & $\mathrm{LC}$ & & & END & $\begin{array}{c}\text { MZUSP 105166, MZUSP } 109762, \\
\text { UFBA } 4843\end{array}$ \\
\hline $\begin{array}{l}\text { Megaleporinus conirostris } \\
\text { (Steindachner } 1875 \text { ) }\end{array}$ & $\mathrm{LC}$ & & $\mathrm{LC}$ & & & $X$ & MZUSP 63463, UFBA 5038 \\
\hline $\begin{array}{l}\text { Megaleporinus elongatus } \\
\text { (Valenciennes 1850) }\end{array}$ & $\mathrm{NE}$ & & NE & & $\mathrm{X}$ & & MZUSP 2823, MZUSP 28773 \\
\hline $\begin{array}{l}\text { Megaleporinus garmani } \\
\text { (Borodin 1929) }\end{array}$ & DD & & $\mathrm{LC}$ & & & $\mathrm{X}$ & MZUSP 87848, UFBA 5238 \\
\hline $\begin{array}{l}\text { Megaleporinus obtusidens } \\
\text { (Valenciennes 1837) }\end{array}$ & $\mathrm{LC}$ & & $\mathrm{LC}$ & & $X$ & & $\begin{array}{l}\text { UFBA } 200, \text { UFBA } 280, \text { UFBA } 835, \\
\text { UFBA } 836, \text { UFBA } 966, \text { UFBA } 1005\end{array}$ \\
\hline
\end{tabular}




\begin{tabular}{|c|c|c|c|c|c|c|c|}
\hline $\begin{array}{l}\text { Schizodon knerii } \\
\text { (Steindachner 1875) }\end{array}$ & $\mathrm{NE}$ & & $\mathrm{NE}$ & & $\mathrm{X}$ & & MZUSP 54676; MCP 16747, UFBA 6582 \\
\hline \multicolumn{8}{|l|}{ Bryconidae } \\
\hline Brycon devillei (Castelnau 1855) & $\mathrm{EN}$ & B1 ab(i,iii) & $\mathrm{EN}$ & B1 ab(i,iii) & & $\mathrm{X}$ & MNHN 4517 \\
\hline Brycon orthotaenia Günther 1864 & $\mathrm{LC}$ & & $\mathrm{LC}$ & & $\mathrm{X}$ & & MZUSP 70220, MCP 16676 \\
\hline Brycon vonoi Lima 2017 & $\mathrm{NE}$ & & $\mathrm{NE}$ & & & $\mathrm{X}$ & MCZ 2416 \\
\hline $\begin{array}{l}\text { Henochilus wheatlandii } \\
\text { Garman } 1890\end{array}$ & $\mathrm{NE}$ & & $\mathrm{NE}$ & & $\mathrm{X}$ & & HU-Zoo 21109 \\
\hline \multicolumn{8}{|l|}{ Characidae } \\
\hline $\begin{array}{l}\text { Astyanax aff. bimaculatus } \\
\text { (Linnaeus 1758) }\end{array}$ & $\mathrm{LC}$ & & $\mathrm{LC}$ & & & $\mathrm{X}$ & $\begin{array}{l}\text { MZUSP 54839, MZUSP 54840, MCP } \\
\text { 17920, MCP 36779, MBML 6407, } \\
\text { MBML 4055, MHNCI-Peixes } 11468\end{array}$ \\
\hline $\begin{array}{l}\text { Astyanax brucutu Zanata, Lima, } \\
\text { di Dario \& Gerhard } 2017\end{array}$ & $\mathrm{NE}$ & & $\mathrm{NE}$ & & & END & $\begin{array}{l}\text { ZUEC 12765, MZUSP 120743, UFBA } \\
\qquad 8167\end{array}$ \\
\hline $\begin{array}{l}\text { Astyanax burgerai Zanata \& } \\
\text { Camelier } 2009\end{array}$ & DD & & DD & & & END & MZUSP 101245, UFBA 4346 \\
\hline $\begin{array}{l}\text { Astyanax epiagos Zanata \& } \\
\text { Camelier } 2008\end{array}$ & DD & & DD & & & END & MZUSP 89568, UFBA 2792 \\
\hline Astyanax lacustris (Lütken 1875) & LC & & $\mathrm{LC}$ & & $\mathrm{X}$ & & $\begin{array}{l}\text { MZUSP 58840, MZUSP } 80125, \\
\text { MZUSP } 80128\end{array}$ \\
\hline $\begin{array}{l}\text { Astyanax lorien Zanata, Burger } \\
\text { \& Camelier } 2018\end{array}$ & $\mathrm{NE}$ & & $\mathrm{NE}$ & & & END & $\begin{array}{c}\text { MZUSP } 123398, \text { UFBA } 5393, \text { UFBA } \\
8109, \text { UFBA } 8359\end{array}$ \\
\hline $\begin{array}{l}\text { Astyanax pelecus } \\
\text { Bertaco \& Lucena } 2006\end{array}$ & LC & & $\mathrm{LC}$ & & & END & UFBA 5634, UFBA 5638 \\
\hline Astyanax rivularis (Lütken 1875) & LC & & $\mathrm{LC}$ & & $\mathrm{X}$ & $\mathrm{X}$ & UFBA 246 \\
\hline $\begin{array}{l}\text { Astyanax rupestris } \\
\text { Zanata, Burger \& Camelier } 2018\end{array}$ & $\mathrm{NE}$ & & $\mathrm{NE}$ & & & END & $\begin{array}{c}\text { MZUSP 89567, MZUSP } 38537, \text { MCP } \\
\text { 53156, UFBA } 2789\end{array}$ \\
\hline $\begin{array}{l}\text { Astyanax sincora } \\
\text { Burger, Carvalho \& Zanata } 2019\end{array}$ & $\mathrm{NE}$ & & $\mathrm{NE}$ & & & END & $\begin{array}{c}\text { MZUSP 120747, MNRJ 48346, UFBA } \\
8201\end{array}$ \\
\hline $\begin{array}{l}\text { Astyanax varii Zanata, Burger, } \\
\text { Vita \& Camelier } 2019\end{array}$ & $\mathrm{NE}$ & & $\mathrm{NE}$ & & & END & $\begin{array}{c}\text { MZUSP 121062, MCP 54205, UFBA } \\
7046\end{array}$ \\
\hline $\begin{array}{l}\text { Astyanax vermilion } \\
\text { Zanata \& Camelier } 2009\end{array}$ & $\mathrm{LC}$ & & $\mathrm{LC}$ & & & END & MZUSP 101243, UFBA 4343 \\
\hline $\begin{array}{l}\text { Compsura heterura } \\
\text { Eigenmann } 1915\end{array}$ & LC & & $\mathrm{LC}$ & & $\mathrm{X}$ & $\mathrm{X}$ & $\begin{array}{c}\text { MZUSP 54626, MZUSP 58801, UFBA } \\
\text { 3005, UFBA } 3301\end{array}$ \\
\hline $\begin{array}{l}\text { Hasemania piatan } \\
\text { Zanata \& Serra } 2010\end{array}$ & $\mathrm{EN}$ & B1 ab(iii,v) & EN & B1 ab(iii,v) & & END & $\begin{array}{c}\text { MZUSP 104538, MZUSP } 104539, \\
\text { UFBA } 4298\end{array}$ \\
\hline
\end{tabular}




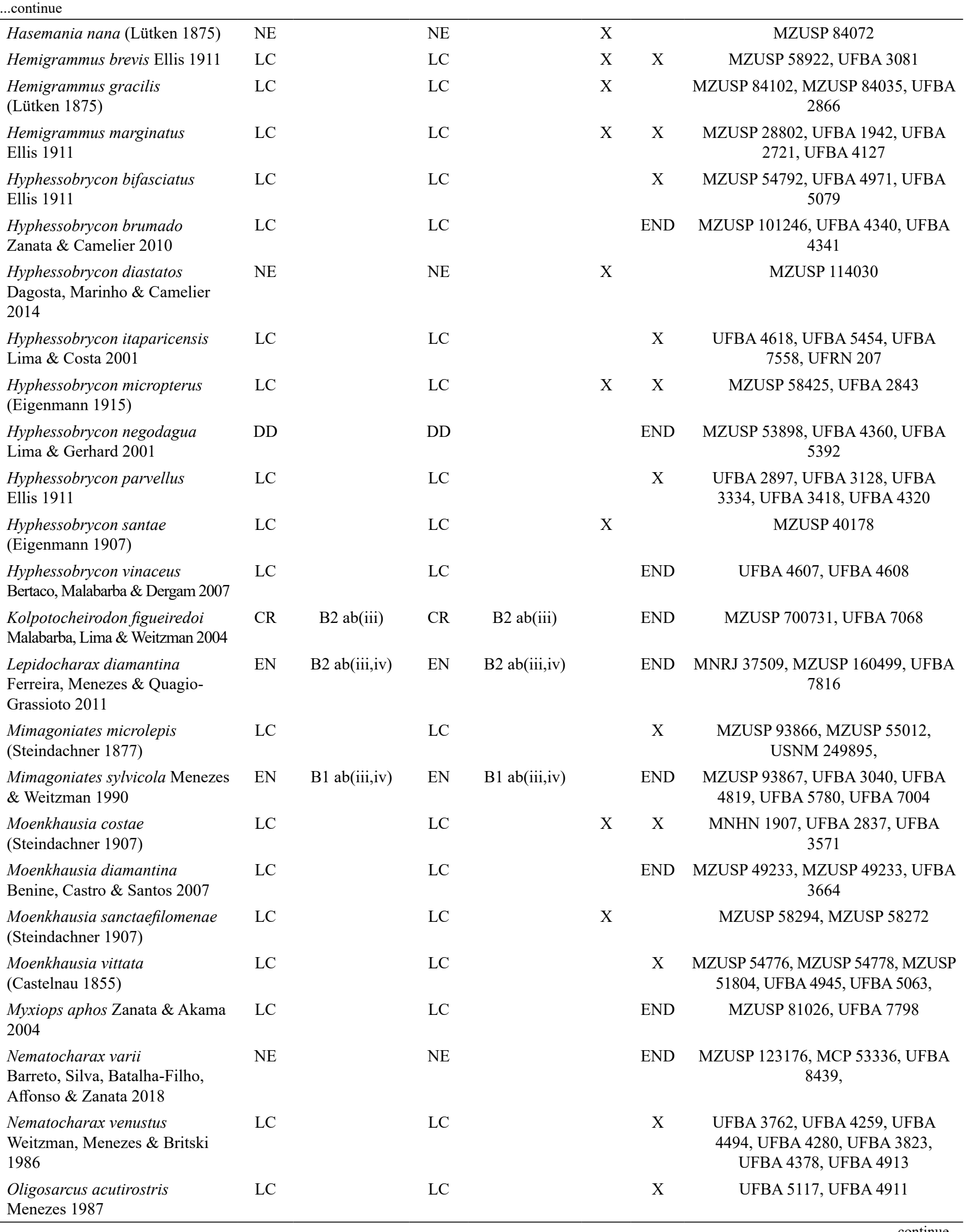




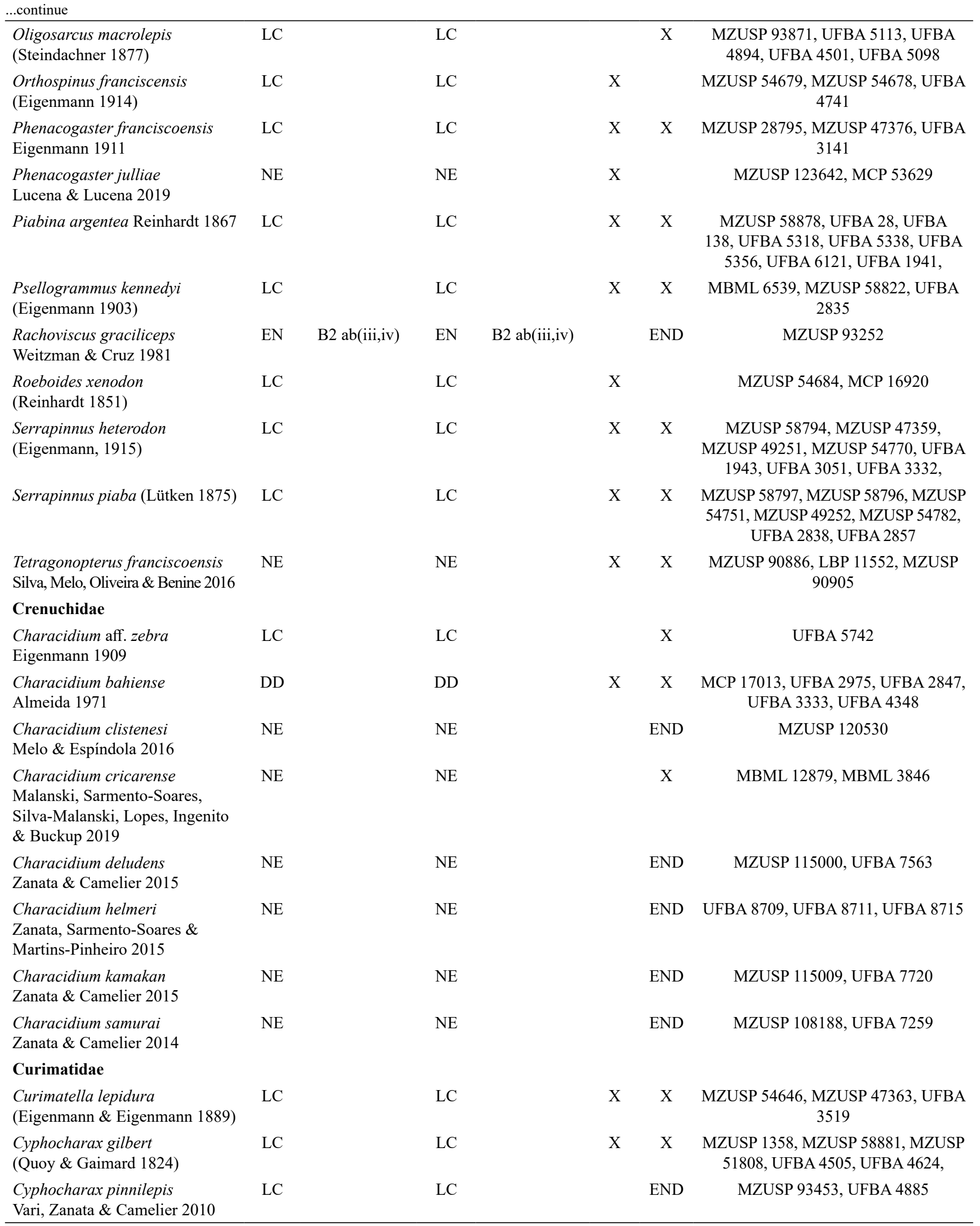


...continue

Steindachnerina elegans

(Steindachner 1875)

LC

LC

\section{Erythrinidae}

Erythrinus kessleri

Steindachner 1877

Hoplerythrinus unitaeniatus

(Spix \& Agassiz 1829)

Hoplias brasiliensis

(Spix \& Agassiz 1829)

Hoplias intermedius

(Günther 1864)

Hoplias malabaricus

(Bloch 1794)

\section{Iguanodectidae}

Bryconops affinis

(Günther 1864)

\section{Lebiasinidae}

Nannostomus beckfordi

Günther 1872

\section{Parodontidae}

Apareiodon hasemani

Eigenmann 1916

Apareiodon itapicuruensis

Eigenmann \& Henn 1916

Parodon hilarii Reinhardt 1867

\section{Prochilodontidae}

Prochilodus argenteus

Spix \& Agassiz 1829

Prochilodus brevis

Steindachner 1875

Prochilodus costatus

Valenciennes 1850

Prochilodus hartii

Steindachner 1875

Prochilodus vimboides

Kner 1859

\section{Serrasalmidae}

Colossoma macropomum

(Cuvier 1816)

Metynnis maculatus (Kner 1858)

Myleus micans (Lütken 1875) LC

Pygocentrus piraya (Cuvier 1819)

Serrasalmus brandtii Lütken, 1875
DD

LC

LC

LC

$\mathrm{LC}$

LC

DD

LC

LC

LC

$\mathrm{LC}$

$\mathrm{LC}$

LC

LC

LC
DD

DD

LC

LC

LC

LC

LC

LC

LC

LC

LC

LC

LC

LC

LC

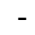

$\mathrm{LC}$

LC

LC
X X MZUSP 58290, MZUSP 54697, MZUSP 54700, UFBA 3417, UFBA 3230, UFBA 3328, UFBA 3209, UFBA 4848, UFBA 4447, UFBA 4247, UFBA 4377

END MZUSP 93891, MZUSP 44018

X X MZUSP 57392, MZUSP 38539, MZUSP 2801, UFBA 2899, UFBA 2966, UFBA 3044

X MZUSP 45483, MZUSP 40174, MZUSP 40170, MZUSP 40270, UFBA 5429

X MZUSP 94050, MZUSP 94434

X X MZUSP 62574, MZUSP 84071, UFBA 3020, UFBA 384, UFBA 3104, UFBA 2947, UFBA 1985, UFBA 3839 , UFBA 3717, UFBA 4916, UFBA 4808

X

MZUSP 57922, MZUSP 54641, MZUSP 57924

X UFBA 1855, UFBA 1887, UFBA 1890, UFBA 1897, UFBA 3742, UFBA 4675, UFBA 4680, UFBA 7412

X MCP 17045

END

UFBA 0234, UFBA 4156

$\mathrm{X}$

MZUSP 58286, MZUSP 28780

$\mathrm{X}$

MZUSP 28778, MZUSP 2040

X UFBA 3436, UFBA 1947, UFBA

5669, UFBA 4628, UFBA 4629

X

X

MZUSP 54756, MZUSP 21549, UFBA 2825

X

MZUSP 42677, UFBA 5272

X UFBA 5094, UFBA 5061, UFBA 5181

NNA

Bezerra et al. 2008

NNA Rodrigues et al. 2018; MCP 36973

NNA

X $\quad \mathrm{M} \quad$ MZUSP 58277, MZUSP 20460

$X \quad$ NNA

MZUSP 57552,

$\mathrm{X}$

$\mathrm{X}$
MZUSP 58296, MZUSP 57550, MZUSP 49254, MZUSP 57508, UFBA 3018, UFBA 2823 


\begin{tabular}{|c|c|c|c|c|c|}
\hline \multicolumn{6}{|l|}{ Triportheidae } \\
\hline $\begin{array}{l}\text { Triportheus signatus } \\
\text { (Garman 1890) }\end{array}$ & $\mathrm{DD}$ & $\mathrm{LC}$ & & $\mathrm{X}$ & UFBA 3173 , UFBA 4836 \\
\hline \multicolumn{6}{|l|}{ Gymnotidae } \\
\hline $\begin{array}{l}\text { Gymnotus bahianus } \\
\text { Campos-da-Paz \& Costa } 1996\end{array}$ & $\mathrm{LC}$ & $\mathrm{LC}$ & & END & $\begin{array}{c}\text { MZUSP 102453, MZUSP } 48949, \\
\text { UFBA 4452, }\end{array}$ \\
\hline $\begin{array}{l}\text { Gymnotus capitimaculatus } \\
\text { Rangel-Pereira } 2014\end{array}$ & $\mathrm{NE}$ & $\mathrm{NE}$ & & END & UFRJ 9785, UFRJ 9964 \\
\hline $\begin{array}{l}\text { Gymnotus interruptus } \\
\text { Rangel-Pereira } 2012\end{array}$ & DD & DD & & END & UFRJ 8218 \\
\hline \multicolumn{6}{|l|}{ Hypopomidae } \\
\hline $\begin{array}{l}\text { Brachyhypopomus menezesi } \\
\text { Crampton, Santana, Waddell \& } \\
\text { Lovejoy } 2016\end{array}$ & $\mathrm{NE}$ & $\mathrm{NE}$ & $\mathrm{X}$ & & MZUSP 87147, MZUSP 40190 \\
\hline \multicolumn{6}{|l|}{ Sternopygidae } \\
\hline $\begin{array}{l}\text { Eigenmannia besouro } \\
\text { Peixoto \& Wosiacki } 2016\end{array}$ & $\mathrm{NE}$ & $\mathrm{NE}$ & END & & MZUSP 57890 \\
\hline \multicolumn{6}{|l|}{ Auchenipteridae } \\
\hline $\begin{array}{l}\text { Glanidium botocudo } \\
\text { Sarmento-Soares \& Martins- } \\
\text { Pinheiro } 2013\end{array}$ & DD & $\mathrm{DD}$ & & $\mathrm{X}$ & MNRJ 32538 \\
\hline $\begin{array}{l}\text { Pseudauchenipterus affinis } \\
\text { (Steindachner 1877) }\end{array}$ & $\mathrm{LC}$ & $\mathrm{LC}$ & & $\mathrm{X}$ & MZUSP 51762, MZUSP 51750 \\
\hline $\begin{array}{l}\text { Pseudauchenipterus jequitinhonhae } \\
\text { (Steindachner 1877) }\end{array}$ & $\mathrm{LC}$ & $\mathrm{LC}$ & & $\mathrm{X}$ & MZUSP 51735, UFBA 05398 \\
\hline Pseudotatia parva Mess 1974 & $\mathrm{NE}$ & $\mathrm{NE}$ & END & & FMNH 70580 \\
\hline $\begin{array}{l}\text { Tatia bockmanni } \\
\text { (Sarmento-Soares \& Buckup 2005) }\end{array}$ & DD & $\mathrm{LC}$ & $\mathrm{X}$ & & MZUSP 82351 \\
\hline $\begin{array}{l}\text { Trachelyopterus galeatus } \\
\text { (Linnaeus } 1766 \text { ) }\end{array}$ & $\mathrm{LC}$ & $\mathrm{LC}$ & $X$ & $X$ & $\begin{array}{c}\text { MZUSP 90280, UFBA 93, UFBA } \\
\text { 130, UFBA 163, UFBA 848, UFBA } \\
\text { 872, UFBA 2819, UFBA } 3046, \text { UFBA } \\
\text { 05119, UFBA } 6648\end{array}$ \\
\hline $\begin{array}{l}\text { Trachelyopterus striatulus } \\
\text { (Steindachner 1877) }\end{array}$ & $\mathrm{LC}$ & $\mathrm{LC}$ & & $X$ & $\begin{array}{c}\text { MZUSP 52627, MZUSP 93912, UFBA } \\
\text { 4712, UFBA 4992, }\end{array}$ \\
\hline \multicolumn{6}{|l|}{ Callichthyidae } \\
\hline $\begin{array}{l}\text { Aspidoras kiriri Oliveira, Zanata, } \\
\text { Tencatt \& Britto } 2017\end{array}$ & $\mathrm{NE}$ & $\mathrm{NE}$ & & END & MNRJ 47400, UFBA 7352 \\
\hline
\end{tabular}




\begin{tabular}{|c|c|c|c|c|c|c|c|}
\hline $\begin{array}{l}\text { Aspidoras maculosus } \\
\text { Nijssen \& Isbrücker } 1976\end{array}$ & $\mathrm{DD}$ & & $\mathrm{DD}$ & & & END & MZUSP 88170, UFBA 3291 \\
\hline $\begin{array}{l}\text { Aspidoras psammatides } \\
\text { Britto, Lima \& Santos } 2005\end{array}$ & DD & & DD & & & END & MZUSP 67194 \\
\hline $\begin{array}{l}\text { Aspidoras virgulatus } \\
\text { Nijssen \& Isbrücker } 1980\end{array}$ & $\mathrm{LC}$ & & $\mathrm{LC}$ & & & $\mathrm{X}$ & MZUSP 39124, MBML 2030 \\
\hline $\begin{array}{l}\text { Callichthys callichthys } \\
\text { (Linnaeus 1758) }\end{array}$ & $\mathrm{LC}$ & & $\mathrm{LC}$ & & $X$ & $\mathrm{X}$ & $\begin{array}{c}\text { UFBA } 159 \text {, UFBA } 2650, \text { UFBA } 3422, \\
\text { UFBA } 5262,\end{array}$ \\
\hline $\begin{array}{l}\text { Corydoras costai Ottoni, } \\
\text { Barbosa \& Katz } 2016\end{array}$ & NE & & $\mathrm{NE}$ & & $\mathrm{X}$ & & UFRJ 7790, MCP 48169 \\
\hline Corydoras garbei Ihering 1911 & $\mathrm{LC}$ & & $\mathrm{LC}$ & & $\mathrm{X}$ & & MZUSP 5319, MZUSP 5324 \\
\hline $\begin{array}{l}\text { Corydoras lacerdai } \\
\text { Hieronimus } 1995\end{array}$ & EN & B1 ab(iii) & EN & B1 ab(iii) & & END & MZUSP 47682, MZUSP 47683 \\
\hline $\begin{array}{l}\text { Corydoras lymnades } \\
\text { Tencatt, Vera-Alcaraz, Britto \& } \\
\text { Pavanelli } 2013\end{array}$ & DD & & $\mathrm{LC}$ & & $\mathrm{X}$ & & MNRJ 22370 \\
\hline $\begin{array}{l}\text { Corydoras multimaculatus } \\
\text { Steindachner } 1907\end{array}$ & $\mathrm{LC}$ & & $\mathrm{LC}$ & & END & & MZUSP 57405, MZUSP 57404 \\
\hline $\begin{array}{l}\text { Corydoras nattereri } \\
\text { Steindachner } 1876\end{array}$ & $\mathrm{LC}$ & & $\mathrm{LC}$ & & & $\mathrm{X}$ & $\begin{array}{l}\text { MZUSP 51796, UFBA 4728, UFBA } \\
2839\end{array}$ \\
\hline $\begin{array}{l}\text { Hoplosternum littorale } \\
\text { (Hancock 1828) }\end{array}$ & $\mathrm{LC}$ & & $\mathrm{LC}$ & & $X$ & $\mathrm{X}$ & MZUSP 57551, UFBA 1611 \\
\hline $\begin{array}{l}\text { Scleromystax prionotos } \\
\text { (Nijssen \& Isbrücker 1980) }\end{array}$ & $\mathrm{LC}$ & & $\mathrm{LC}$ & & & $\mathrm{X}$ & $\begin{array}{l}\text { MCP 23650, MBML 1470, MBML } \\
\text { 1546, UFBA } 5073\end{array}$ \\
\hline \multicolumn{8}{|l|}{ Clariidae } \\
\hline Clarias gariepinus (Burchell 1822) & - & & - & & & NNA & Rocha et al 2008, MNRJ 28363 \\
\hline \multicolumn{8}{|l|}{ Doradidae } \\
\hline $\begin{array}{l}\text { Franciscodoras marmoratus } \\
\text { (Lütken 1874) }\end{array}$ & LC & & $\mathrm{LC}$ & & $\mathrm{X}$ & & MZUSP 2201, UFBA 208 \\
\hline $\begin{array}{l}\text { Kalyptodoras bahiensis Higuchi, } \\
\text { Britski \& Garavello } 1990\end{array}$ & EN & B2 ab(iii) & EN & B2 ab(iii) & & END & $\begin{array}{c}\text { MZUSP } 38565 \text {, UFBA } 3171, \text { UFBA } \\
\text { 7108, UFBA } 7455\end{array}$ \\
\hline $\begin{array}{l}\text { Wertheimeria maculata } \\
\text { Steindachner } 1877\end{array}$ & $\mathrm{LC}$ & & $\mathrm{LC}$ & & & $\mathrm{X}$ & UFBA 5667 \\
\hline \multicolumn{8}{|l|}{ Heptapteridae } \\
\hline $\begin{array}{l}\text { Acentronichthys leptos } \\
\text { Eigenmann \& Eigenmann } 1889\end{array}$ & EN & B1 ab(iii) & $\mathrm{LC}$ & & & $\mathrm{X}$ & MZUSP 93856, UFBA 6019 \\
\hline $\begin{array}{l}\text { Cetopsorhamdia iheringi } \\
\text { Schubart \& Gomes } 1959\end{array}$ & $\mathrm{LC}$ & & $\mathrm{LC}$ & & $\mathrm{X}$ & $\mathrm{X}$ & UFBA 4370, UFBA 5121, UFBA 6097 \\
\hline $\begin{array}{l}\text { Imparfinis borodini } \\
\text { Mees \& Cala } 1989\end{array}$ & NE & & $\mathrm{NE}$ & & $\mathrm{X}$ & $\mathrm{X}$ & MBML 10327, MBML 10729 \\
\hline $\begin{array}{l}\text { Imparfinis minutus } \\
\text { (Lütken 1874) }\end{array}$ & $\mathrm{LC}$ & & $\mathrm{LC}$ & & $\mathrm{X}$ & $\mathrm{X}$ & $\begin{array}{c}\text { UFBA } 935, \text { UFBA 4958, UFBA 5071, } \\
\text { UFBA 4987, UFBA } 5053\end{array}$ \\
\hline $\begin{array}{l}\text { Phenacorhamdia tenebrosa } \\
\text { (Schubart 1964) }\end{array}$ & DD & & $\mathrm{LC}$ & & & $\mathrm{X}$ & MCP 36935, MCP 36723, UFBA 7114 \\
\hline $\begin{array}{l}\text { Pimelodella harttii } \\
\text { (Steindachner 1877) }\end{array}$ & DD & & DD & & & $\mathrm{X}$ & MZSUP 5141, UFBA 5647 \\
\hline $\begin{array}{l}\text { Pimelodella itapicuruensis } \\
\text { Eigenmann } 1917\end{array}$ & DD & & $\mathrm{LC}$ & & & $\mathrm{X}$ & MZUSP 88169 \\
\hline $\begin{array}{l}\text { Pimelodella lateristriga } \\
\text { (Lichtenstein 1823) }\end{array}$ & $\mathrm{LC}$ & & $\mathrm{LC}$ & & & $\mathrm{X}$ & $\begin{array}{c}\text { MZUSP 93864, MZUSP 54763, } \\
\text { MZUSP 51798, UFBA 4973, UFBA } \\
4984,\end{array}$ \\
\hline $\begin{array}{l}\text { Rhamdia enfurnada } \\
\text { Bichuette \& Trajano } 2005\end{array}$ & $\mathrm{LC}$ & & $\mathrm{LC}$ & & END & & LIRP 5643 \\
\hline
\end{tabular}


...continue

\section{Rhamdia jequitinhonha}

Silfvergrip 1996

Rhamdia quelen (Quoy \&

Gaimard 1824)

(Eigenmann \& Eigenmann 1888)
Rhamdiopsis krugi

Bockmann \& Castro 2011

Rhamdiopsis sp.

\section{Loricariidae}

Harttia longipinna Langeani, Oyakawa \& Montoya-Burgos 2001

Hirtella carinata Pereira, Zanata, Cetra \& Reis 2014

Hypostomus brevicauda (Günther 1864)

Hypostomus francisci (Lütken 1874)

Hypostomus jaguar Zanata, Sardeiro \& Zawadzki 2013

Hypostomus johnii

(Steindachner 1877)

Hypostomus leucophaeus

Zanata \& Pitanga 2016

Hypostomus lima (Lütken 1874)

Hypostomus macrops

Hypostomus unae

(Steindachner 1878)

Hypostomus vaillanti

(Steindachner 1877)

Hypostomus velhochico

Zawadzki, Oyakawa, Britski 2017

Hypostomus wuchereri

(Günther 1864)

Megalancistrus barrae

(Steindachner 1910)

Otocinclus xakriaba

Schaefer 1997

Otothyris travassosi Garavello, Britski \& Schaefer 1998

Pareiorhaphis bahianus

(Gosline 1947)

Pareiorhaphis lophia Pereira \&

Zanata 2014

Parotocinclus adamanteus 2019

Parotocinclus arandai

LC

VU

VU

DD

NE

DD

LC

LC

NE

NE

NE

$\mathrm{NE}$

DD

NE

NE

DD

LC

LC

LC

LC

NE

Sarmento-Soares, Lehmann \& Martins-Pinheiro 2009

VU

LC

$$
\text { B1ab(iii) }
$$

VU

B1ab(iii)

D2

VU

DD

NE

DD

LC

LC

NE

NE

$\mathrm{NE}$

$\mathrm{NE}$

LC

DD

NE

DD

LC

LC

LC

LC

NE

LC
MZUSP 102718

X X

MCP 16658, MZUSP 83796, MZUSP 93851 MZUSP 54006, MZUSP 101358, UFBA 3006, UFBA 2894, UFBA 03353,UFBA 4263, UFBA 4858, , UFBA 4909, UFBA 4956

END LIRP 5929, LIRP 5931, LIRP 5930

D2 END

M. E. Bichuette (comm. pers.)

DZSJRP003666, MZUSP 57168

END

END

ANSP 198032, MCP 48127, UFBA 5655

X

BMNH1864

X

UFBA 4191, UFBA 6641

END

X

MCZ 7863, NMW 44192

END

MZUSP 119822, UFBA 2993

$\mathrm{X}$

$\mathrm{X}$

UFBA 2046

MBML 11304, MBML- 10813, NUP 20383

X

BMNH 1861, NMW 44259

X

NMW 44273

X

MCP 16689, MZUSP 94586, MZUSP 120452

X

BMNH1863

END

NMW 48019

X

MZUSP 51103, MCP 16877

X

MZUSP 94020, MZUSP 51435, MZUSP 94021, MZUSP 39095, MZUSP 51439, MZUSP 51438, MZUSP 51803, UFBA 4931,

END UFBA 4486, UFBA 4555, UFBA 5100

END MCP 47711, MCP 47712, MCP 48004, MZUSP 86089, MZUSP 86154, MZUSP 88163, UFBA 6188, UFBA 7026, UFBA 7063, UFBA 7350

END MZUSP 124560, MCP 54151, MZUSP 93274

END

MBML 2135, MBML 1486 


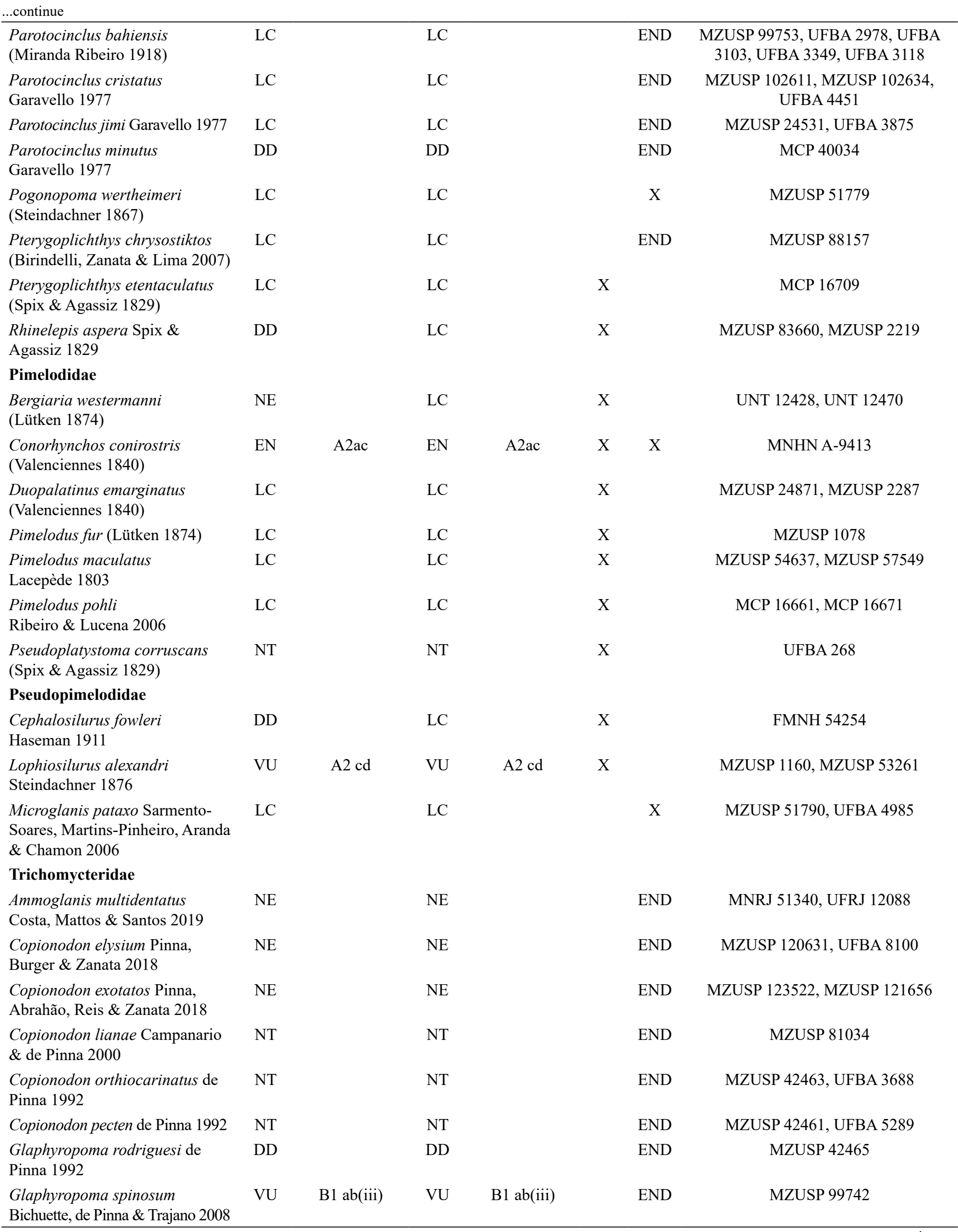


Freshwater fishes of the Bahia State

\begin{tabular}{|c|c|c|c|c|c|c|c|}
\hline $\begin{array}{l}\text { Ituglanis cahyensis Sarmento- } \\
\text { Soares, Martins-Pinheiro, Aranda } \\
\text { \& Chamon } 2006\end{array}$ & $\mathrm{EN}$ & B1 ab(iii) & EN & B1 ab(iii) & & $\mathrm{X}$ & MNRJ 28404, MNRJ 28406 \\
\hline $\begin{array}{l}\text { Ituglanis paraguassuensis } \\
\text { Campos-Paiva \& Costa } 2007\end{array}$ & $\mathrm{DD}$ & & $\mathrm{DD}$ & & & END & MZUSP 63138 \\
\hline $\begin{array}{l}\text { Stegophilus insidiosus } \\
\text { Reinhardt } 1859\end{array}$ & $\mathrm{LC}$ & & $\mathrm{LC}$ & & $X$ & & MBML 9078 \\
\hline $\begin{array}{l}\text { Trichomycterus bahianus } \\
\text { Costa } 1992\end{array}$ & $\mathrm{LC}$ & & $\mathrm{LC}$ & & & END & MZUSP 38636, MBML 1580 \\
\hline $\begin{array}{l}\text { Trichomycterus pradensis } \\
\text { Sarmento-Soares, Martins- } \\
\text { Pinheiro, Aranda \& Chamon } 2005\end{array}$ & $\mathrm{LC}$ & & $\mathrm{LC}$ & & & $\mathrm{X}$ & MBML 1480, MBML 1520 \\
\hline $\begin{array}{l}\text { Trichomycterus rubbioli } \\
\text { Bichuette \& Rizzato } 2012\end{array}$ & VU & D2 & VU & D2 & END & & $\begin{array}{l}\text { MZUSP 110977, MZUSP } 110978, \\
\text { MZUSP } 110984\end{array}$ \\
\hline $\begin{array}{l}\text { Trichomycterus tete Barbosa \& } \\
\text { Costa } 2011\end{array}$ & $\mathrm{LC}$ & & $\mathrm{LC}$ & & & END & $\begin{array}{c}\text { UFRJ 7774, UFRJ 7775, UFRJ 7776, } \\
\text { UFRJ } 8082\end{array}$ \\
\hline \multicolumn{8}{|l|}{ CYPRINODONTIFORMES } \\
\hline \multicolumn{8}{|l|}{ Poeciliidae } \\
\hline $\begin{array}{l}\text { Pamphorichthys hollandi } \\
\text { (Henn 1916) }\end{array}$ & $\mathrm{LC}$ & & $\mathrm{LC}$ & & $\mathrm{X}$ & $\mathrm{X}$ & $\begin{array}{c}\text { MZUSP 58869, MZUSP 57507, UFBA } \\
\text { 2892, UFBA } 3037,\end{array}$ \\
\hline $\begin{array}{l}\text { Poecilia vivipara } \\
\text { Bloch \& Schneider } 1801\end{array}$ & $\mathrm{LC}$ & & $\mathrm{LC}$ & & $\mathrm{X}$ & $\mathrm{X}$ & $\begin{array}{c}\text { MZUSP 93991, MZUSP 51810, UFBA } \\
\text { 3021, UFBA 2860, UFBA 3331, } \\
\text { UFBA 3121, UFBA 4165, UFBA } \\
\text { 4257, UFBA 4239, UFBA 4382, }\end{array}$ \\
\hline \multicolumn{8}{|l|}{ Rivulidae } \\
\hline $\begin{array}{l}\text { Anablepsoides bahianus } \\
\text { (Huber 1990) }\end{array}$ & $\mathrm{DD}$ & & $\mathrm{DD}$ & & & END & UFBA 3423, UFBA 2951 \\
\hline $\begin{array}{l}\text { Atlantirivulus depressus } \\
\text { (Costa 1991) }\end{array}$ & NT & & NT & & & END & UFPB 2213, UFRJ 2118 \\
\hline $\begin{array}{l}\text { Atlantirivulus unaensis } \\
\text { (Costa \& de Luca 2009) }\end{array}$ & $\mathrm{DD}$ & & $\mathrm{DD}$ & & & END & UFRJ 6597 \\
\hline Cynolebias altus Costa 2001 & $\mathrm{LC}$ & & $\mathrm{LC}$ & & END & & MZUSP 62564, MZUSP 62565 \\
\hline $\begin{array}{l}\text { Cynolebias attenuatus } \\
\text { Costa } 2001\end{array}$ & DD & & DD & & END & & MZUSP 62566, MZUSP 62567 \\
\hline Cynolebias elegans Costa 2017 & $\mathrm{NE}$ & & $\mathrm{NE}$ & & END & & UFRJ 9431, UFRJ 6890 \\
\hline Cynolebias gibbus Costa 2001 & DD & & DD & & END & & MZUSP 62568, UFRJ 4796 \\
\hline Cynolebias gilbertoi Costa 1998 & $\mathrm{DD}$ & & $\mathrm{DD}$ & & END & & MZUSP 52304, MZUSP 52305 \\
\hline $\begin{array}{l}\text { Cynolebias itapicuruensis } \\
\text { Costa } 2001\end{array}$ & $\mathrm{DD}$ & & DD & & & END & UFBA 2626 \\
\hline
\end{tabular}




\begin{tabular}{|c|c|c|c|c|c|c|c|}
\hline Cynolebias obscurus Costa 2014 & NE & & $\mathrm{NE}$ & & END & & UFRJ 6774 \\
\hline Cynolebias oticus Costa 2014 & $\mathrm{NE}$ & & $\mathrm{NE}$ & & END & & UFRJ 9437 \\
\hline $\begin{array}{l}\text { Cynolebias paraguassuensis } \\
\text { Costa, Suzart \& Nielsen } 2007\end{array}$ & $\mathrm{DD}$ & & $\mathrm{DD}$ & & & END & UFRJ 6454, UFRJ 6455 \\
\hline Cynolebias rectiventer Costa 2014 & $\mathrm{NE}$ & & $\mathrm{NE}$ & & END & & UFRJ 8896 \\
\hline Cynolebias roseus Costa 2014 & $\mathrm{NE}$ & & $\mathrm{NE}$ & & END & & UFRJ 9330, UFRJ 9331 \\
\hline $\begin{array}{l}\text { Cynolebias vazabarrisensis } \\
\text { Costa } 2001\end{array}$ & DD & & DD & & & END & MZUSP 62561, MZUSP 62560 \\
\hline Hypsolebias caeruleus Costa 2013 & $\mathrm{NE}$ & & $\mathrm{LC}$ & & END & & UFRJ 6855 \\
\hline $\begin{array}{l}\text { Hypsolebias faouri Britzke, } \\
\text { Nielsen \& Oliveira } 2016\end{array}$ & NE & & $\mathrm{NE}$ & & END & & ZUEC 10796 \\
\hline Hypsolebias flagellatus (Costa 2003) & $\mathrm{NE}$ & & NA & & $\mathrm{X}$ & & MCP 28578, UFRJ 4788 \\
\hline $\begin{array}{l}\text { Hypsolebias fulminantis } \\
\text { (Costa \& Brasil 1993) }\end{array}$ & $\mathrm{CR}$ & B2ab(i,ii,iii,iv) & $\mathrm{CR}$ & B2ab(i,ii,iii,iv) & END & & MZUSP 43674, UFRJ 685 \\
\hline $\begin{array}{l}\text { Hypsolebias gardneri Costa, } \\
\text { Amorim \& Mattos } 2018\end{array}$ & $\mathrm{NE}$ & & $\mathrm{NE}$ & & $\mathrm{X}$ & & UFRJ 11859 \\
\hline Hypsolebias igneus (Costa 2000) & $\mathrm{CR}$ & B2ab(i,ii,iii,iv) & $\mathrm{CR}$ & B2ab(i,ii,iii,iv) & END & & MZUSP 56254, UFRJ 4857 \\
\hline $\begin{array}{l}\text { Hypsolebias lopesi (Nielsen, } \\
\text { Shibatta, Suzart \& Martín 2010) }\end{array}$ & $\mathrm{NE}$ & D2 & VU & D2 & END & & MZUSP 103102 \\
\hline $\begin{array}{l}\text { Hypsolebias mediopapillatus } \\
\text { (Costa 2006) }\end{array}$ & VU & D2 & VU & D2 & END & & UFRJ 5406, MCP 40139 \\
\hline Hypsolebias nitens Costa 2012 & $\mathrm{NE}$ & & $\mathrm{LC}$ & & END & & UFRJ 8289 \\
\hline $\begin{array}{l}\text { Hypsolebias nudiorbitatus } \\
\text { Costa } 2011\end{array}$ & $\mathrm{NE}$ & & DD & & & END & UFRJ 6837 \\
\hline Hypsolebias picturatus (Costa 2000) & VU & D2 & VU & D2 & END & & MZUSP 59228, UFRJ 5053 \\
\hline $\begin{array}{l}\text { Hypsolebias pterophyllus } \\
\text { Costa } 2012\end{array}$ & $\mathrm{NE}$ & & $\mathrm{LC}$ & & END & & UFRJ 8376 \\
\hline $\begin{array}{l}\text { Hypsolebias shibattai Nielsen, } \\
\text { Martins, Araujo \& Suzart } 2014\end{array}$ & $\mathrm{NE}$ & & NA & & END & & ZUEC 7648 \\
\hline $\begin{array}{l}\text { Hypsolebias trifasciatus Nielsen, } \\
\text { Martins, Araújo, Lira \& Four } 2014\end{array}$ & $\mathrm{NE}$ & & NA & & END & & ZUEC 8302 \\
\hline $\begin{array}{l}\text { Kryptolebias hermaphroditus } \\
\text { Costa } 2011\end{array}$ & $\mathrm{NE}$ & & $\mathrm{NE}$ & & & $\mathrm{X}$ & UFRN 4344 \\
\hline $\begin{array}{l}\text { Melanorivulus decoratus } \\
\text { (Costa 1989) }\end{array}$ & NT & & NT & & END & & MZUSP 39982, MZUSP 39983 \\
\hline
\end{tabular}




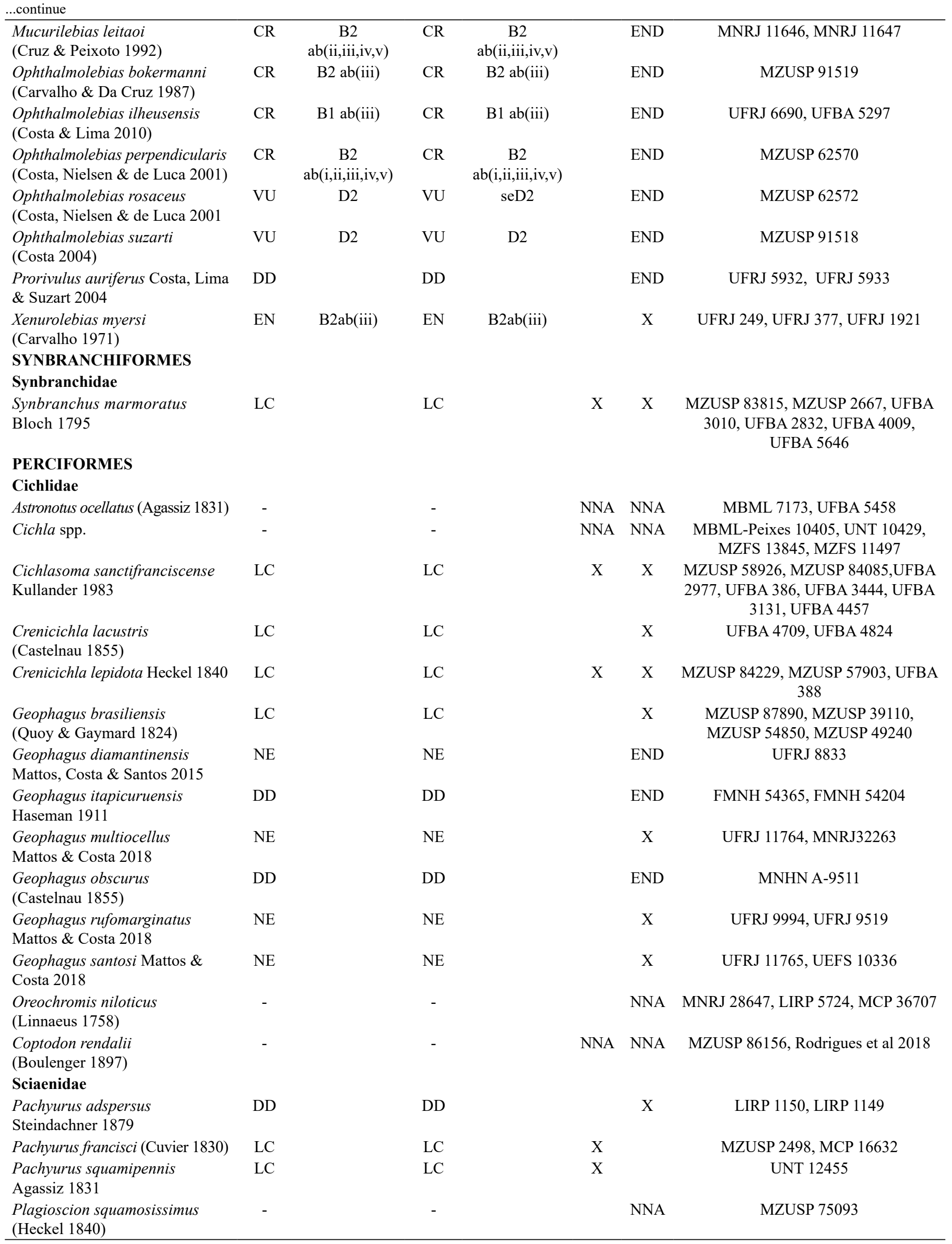


Only $40(14 \%)$ native species are shared between the two ecoregions. Eleven non-native species were listed in Bahia, which accounted for less than $5 \%$ of all species analyzed. Among the 30 families recorded (excluding those related only to non-native species), the most representative were Characidae and Rivulidae, with 53 and 48 species, respectively, followed by Loricariidae with 28 species (Figure 2). Many species were described in the last 20 years, 75 from the NMA and 41 from the SFR, corresponding to $27 \%$ and $15 \%$ of all species listed in this study, respectively (Table 1).

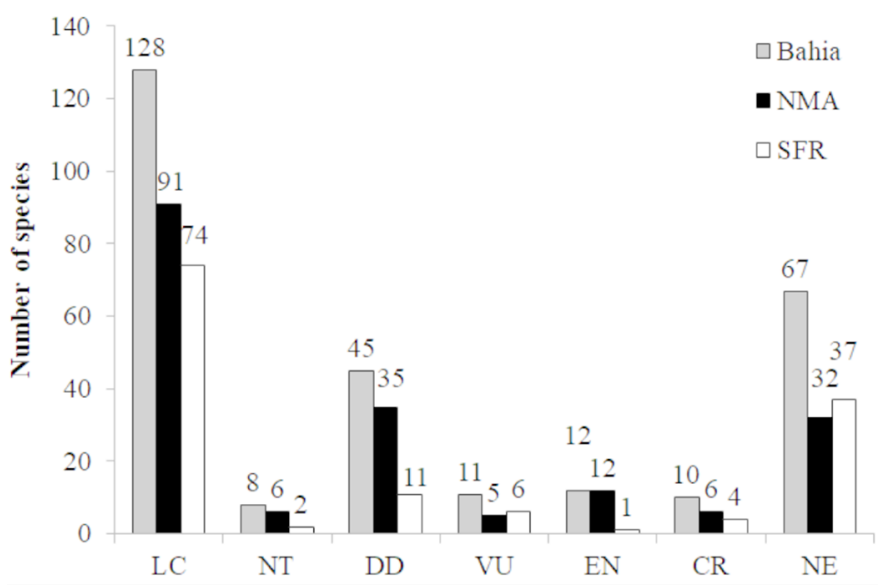

Figure 2. Number of freshwater fish species recorded per family in Bahia State and separately for each ecoregion studied (NMA, Northeastern Mata Atlantica; SFR, São Francisco).

\section{Assessment of conservation status}

In this study, 214 freshwater fish species were evaluated. Most of them were classified as 'Least Concern' (LC), 45 as 'Data Deficient' (DD), and eight as 'Near Threatened' (NT), which are not considered threatened categories according to the IUCN criteria (IUCN 2012) (Table 1, Figure 3). Thirty-three freshwater fish species (26 of them endemic to Bahia) were included in the threatened categories: 11 as 'Vulnerable' (VU), 12 as 'Endangered' (EN), and 10 as 'Critically Endangered' (CR) (Table 1, Figure 3). The Bahia State Red list has been already published by the Secretaria do Meio Ambiente do Estado da Bahia (SEMA 2017), although it contains only the threatened species and their conservation status.

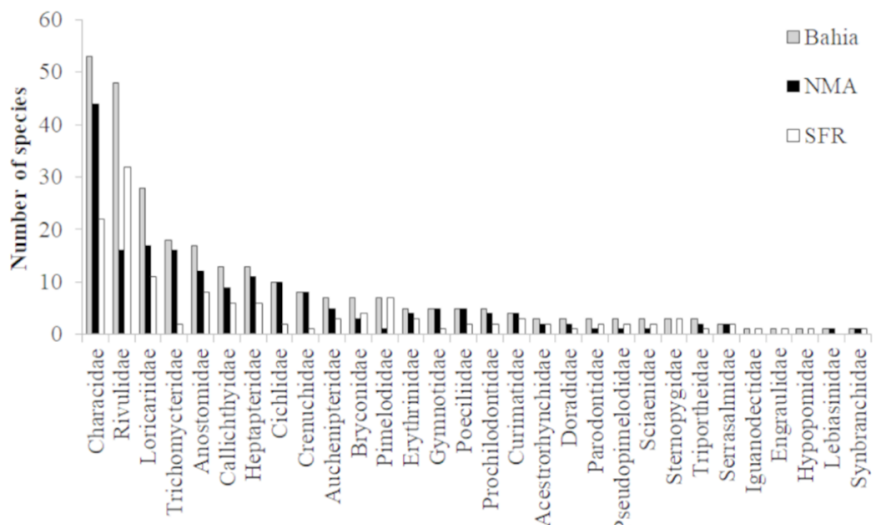

Figure 3. Number of freshwater fish species in Bahia State classified according to the categories proposed by IUCN (2012): LC, least concern; NT, near threatened; $\mathrm{DD}$, data deficient; VU, vulnerable; EN, endangered; CR, critically endangered. NMA, Northeastern Mata Atlantica freshwater ecoregion; SFR, São Francisco freshwater ecoregion.
Most of the threatened species are in the NMA (23 species, 69.7\%), while $10(30.3 \%)$ endangered species are found in the SFR (Figure 3). Most of these belong to the family Rivulidae, with 14 species $(42.4 \%$ of threatened species), seven in each ecoregion. In addition, several small fish species of the Characidae, Heptapteridae, and Trichomycteridae families were also representative in the list of endangered species. These fishes are typical of first- and second-order streams, which may suffer more intensely from the effects of human activities (Oliveira \& Bennemann 2005). Among tricomicterids and heptaperids, three troglobitic species (Glaphyropoma spinosum, Rhamdiopsis krugi, and Rhamdiopsis sp.) stand out among all others because of their low population density and restricted distribution in some caverns (Bockmann \& Castro 2010, Bichuette et al. 2008, M. E. Bichuette pers. comm.), which are not protected in any conservation unit. Taken together, these factors can chronically devastate these populations and compromise species survival.

\section{Discussion}

\section{Species composition}

In this study, 281 native freshwater fish species were recorded in Bahia State, distributed in coastal drainages of the NMA ecoregion and the São Francisco river basin draining the state. The composition of the Bahia State species partially agrees with the overall pattern of the Brazilian fish fauna, in which most species belong to Characidae, followed by Loricariidae (Bizerril 1994, Gonçalves \& Braga 2012, Camelier \& Zanata 2014a, Ferreira et al. 2014). Curiously, Bahia is one of the Brazilian states with the largest records of Rivulidae in its basins (see Frick \& Eschmeyer 2020). Among the remaining families occurring in the state, more than half are represented by five or fewer species.

The number of Rivulidae species is higher in the SFR ecoregion, mainly due to the presence of several annual fish species of the genera Hypsolebias and Cynolebias that inhabit temporary pools in the tributaries at the right margin of the middle São Francisco river basin (Costa 2014, Costa et al. 2014). In the drainages of the NMA ecoregion, Rivulidae is mainly represented by the annual fish species of the genus Ophthalmolebias, most of them endemic to Bahia, presenting a narrow distribution, usually restricted to a single basin (Costa \& Lima 2010). In this ecoregion, Rivulidae and Loricariidae have almost the same number of species, differing from the general pattern found in the other Brazilian coastal drainages cited above.

The river basins included in the NMA and SFR freshwater ecoregions which draining the state of Bahia exhibit a distinct composition, with only 40 native species shared between them. Most species from the NMA are characterized by a small size, possibly related to the large number of small streams of this ecoregion. According to several authors (e.g., Weitzman \& Vari 1988, Castro 1999, Casatti et al. 2001, Abilhoa et al. 2011), the ichthyofauna of these freshwater ecosystems is mainly composed of small-sized species. In the São Francisco river basin, in addition to small fish species, larger species, most of them important for artisanal fisheries, are found, including migratory species such as Conorhynchos conirostris, Prochilodus argenteus, Pseudoplatystoma corruscans, and Salminus franciscanus (Sato \& Godinho 2003, Godinho \& Kynard 2006). 
However, the number of species gathered in the present study is likely underestimated due to a range of factors. Some undescribed species were not counted, the only exception being Rhamdiopsis sp. from the Chapada Diamantina region (SFR). Some nominal widespread species may represent species complexes that need revisionary studies to solve the confusing taxonomy and the definition of the new species described (e.g., Astyanax bimaculatus, A. fasciatus, Hoplias malabaricus, Geophagus brasiliensis, Rhamdia quelen). In addition, some sub-basins were not sampled (e.g., most tributaries of the middle São Francisco in southwestern Bahia and headwaters of various river basins of the NMA).

More than 20 years ago, Menezes (1996a, b) cited the lack of knowledge about the fish fauna of drainages in Brazil's Northeastern region. Although a series of studies increasing that knowledge were conducted at that time, the results were far from satisfactory and Northeast Brazil is still pointed out as a region of scarce available information about freshwater fish records in a global database compilation (Tedesco et al. 2017). Current evidence of the lack of knowledge about the ichthyofauna in this region is the large number of taxa considered "sp." or accompanied by the terms "cf." or "aff." in taxonomic inventories (e.g., SarmentoSoares et al. 2009a, Cetra et al. 2010, Burger et al. 2011, Ramos et al. 2014). Although numerous freshwater fish species from the Bahia river basins have been described in the last two decades (e.g., Bertaco \& Lucena 2006, Ribeiro \& Lucena 2006, Lima \& Britski 2007, Benine et al. 2007, Zanata \& Camelier 2008, 2009, 2010, 2014, 2015, Bichuette et al. 2008, Sarmento-Soares et al. 2009b, Sarmento-Soares et al. 2011, Ferreira et al. 2014, Vari et al. 2010, Bichuette \& Rizzato 2012, Oliveira et al. 2013, Sarmento-Soares \& Martins-Pinheiro 2013, Camelier \& Zanata 2014b, Zanata et al. 2015, 2017, 2018, Mattos et al. 2015, Peixoto \& Wosiacki 2016, Zanata \& Pitanga 2016, Zawadzki et al., 2017; Barreto et al. 2018, Mattos \& Costa, 2018, Burger et al. 2019, Lucena \& Lucena 2019), ichthyological explorations continue to reveal unknown species, especially in small tributaries and in upper drainage areas. The increase in the number of species recently described from the São Francisco river basin has already been documented in the literature (see Alves et al. 2011 and Barbosa et al. 2017).

\section{Conservation concerns}

Overall, the results of the state and national conservation status assessments were remarkably similar, with divergences only for two species: Acentronichthys leptos and Hypomasticus mormyrops. In Bahia State, both species were included in threatened categories (VU and EN, respectively) since they occur at few impacted localities with continuing decline in the habitat quality. However, these species were classified as LC in the national assessment (MMA 2014) since their total distribution was considered wider, including river basins outside Bahia where these species are more abundant and are not threatened (e.g., Paraíba do Sul, Doce, plus small drainages in the states of São Paulo and Rio de Janeiro).

The conservation status of some species which occur in Bahia State changed in the last years. Some examples are the catfish 'pacamã' Lophiosilurus alexandri and the killifish Hypsolebias adornatus, both endemic to the SFR ecoregion, which were classified before as LC (MMA 2004) are now VU in both lists (SEMA 2017, MMA 2014). Several subpopulations of $L$. alexandri were probably locally extinct, reducing their population size by at least $30 \%$ (ICMBio 2018). On the other hand, L. alexandri was introduced in the Doce river, where became an important resource for artisanal fishing (Alves et al. 2007).
Additionally, this species is cultivated in tanks as a commercial species for food near Colatina, Espírito Santo State (L. M. SarmentoSoares pers. obs.). Nowadays, H. adornatus is also VU because one of its few known localities were destroyed, leading to local extinction. Moreover, this species is much appreciated as an aquarium fish, posing an additional and severe threat to this species. The category of three other annual killifishes, Hypsolebias fulminantis, H. ghisolfi, and Ophthalmolebias perpendicularis changed from VU (MMA 2004) to CR (MMA 2014). The first two, endemic to Bahia (SFR), are sympatric and were commonly found in temporary floodplain pools of Rio das Rãs, a tributary of the São Francisco basin (Costa 2007). However, their distribution is now restricted to a few localities as a consequence of the agricultural development and potteries established in the region. The case of $O$. perpendicularis is even more concerning since the species is currently classified as $\mathrm{CR}$, the highest extinction risk category. In fact, this annual rivulid species may already be extinct since it was only known from the type locality in the Jequitinhonha river basin (NMA ecoregion), which was destroyed, and there has been no record of this species since 2000 (ICMBio 2018).

The large number of threatened freshwater fish species in the NMA ecoregion (23) is possibly due to the remarkable endemism of its ichthyofauna (Bizerril 1994, Ribeiro 2006, Camelier \& Zanata 2014a) associated with the significant human occupation and alteration of the coastal region (Langeani et al. 2009). In the SFR ecoregion, most threatened species are annual killifishes, whose populations are disappearing due to the strong degradation and grounding of their habitats (see Costa 2002, ICMBio 2018). Eight species were classified as NT and may soon move to the list of endangered species if no conservation measures are adopted. In addition to the increasing human exploitation, the relatively small number of conservation units and protected areas in Bahia may be insufficient for the preservation of its fish fauna. Only $11.6 \%$ of the state area is protected as Conservation Units and less than $20 \%$ of these are in the higher protective level (Allen 2015). Additionally, we point out that most of these protect areas act as biodiversity islands, since they are surrounded by diverse monocultures, pastures and urban centers. One way to reduce these negative effect is the adoption of public polices that favor more environmentally appropriate agricultural practices in the buffer zones of Conservation Units, such as agroforestry systems (Sarmento-Soares \& Martins-Pinheiro, 2017; Ewert et al. 2013).

The National Action Plans (Planos de Ação Nacional, PANs, in Portuguese) coordinated by the ICMBio, which have as main mission conserving Brazilian biodiversity, are public policies identifying and guiding priority actions to combat threats that endanger populations of species or environments (Polaz 2014). Therefore, the success of the PAN depends of both taxonomic information and data of the assessment of the conservation status of the species, such as provided in the present study. There are three PAN designed for freshwater fishes whose actions directly affect the conservation of the ichthyofauna in the state of Bahia: (1) Action Plan for the threatened species from the São Francisco watershed (Ordinance ICMBio $n^{\circ}$ 34, 27 May 2015), aiming mainly to improve the knowledge about threatened species and mitigate human impacting activities, to promote the conservation and recovery of aquatic fauna in the São Francisco river in five years (2015-2020); 
(2) Action Plan for the threatened fish species from the Atlantic Forest rivers (Ordinance ICMBio $\mathrm{n}^{\circ} 370,1$ August 2019), objecting to increase the conservation status and popularization of fishes, rivers, and streams of the Atlantic Forest in five years (2019-2024); and (3) Action Plan for the threatened species of Rivulidae family, with the general objective of establishing mechanisms to protect the rivulids and canceling the loss of habitat of the focal species, in five years (2013-2018); the second cycle of this PAN (2020-2025) was approved and it is in preparation, awaiting publication of the new ordinance.

Finally, it is expected that these joint actions (e.g., taxonomic studies, lists of species, conservation status of species, public policies) will contribute not only to the increase of the knowledge of the fish fauna but also to the conservation of these species and the environments inhabited by them.

\section{Acknowledgements}

The authors are grateful to ICMBio for technical support and to Dr. Maria Elina Bichuette (UFSCar) for scientific support with the troglobitic fish species. The 2013 Workshop was financially supported by Instituto Driades.

\section{Authors Contributions}

André Teixeira Silva: Substantial contribution in the concept and design of the study; Contribution to manuscript preparation; Contribution to data analysis and interpretation.

Ricardo Jucá Chagas: Substantial contribution in the concept and design of the study.

Alexandre Clistenes Santos: Contribution to data analysis and interpretation.

Angela Maria Zanata: Contribution to critical revision, adding intellectual content.

Beatriz Kawamura Rodrigues: Contribution to data analysis and interpretation.

Carla Natacha Marcolino Polaz: Contribution to critical revision, adding intellectual content.

Carlos Bernardo Mascarenhas Alves: Contribution to critical revision, adding intellectual content.

Cristiana Souza Vieira: Contribution to data collection.

Fabiane Barreto Souza: Contribution to data collection.

Fábio Vieira: Contribution to critical revision, adding intellectual content.

Francisco Alexandre Costa Sampaio: Contribution to data analysis and interpretation.

Harildon Ferreira: Contribution to data collection.

Hilda Susele Rodrigues Alves: Contribution to data collection.

Luisa Maria Sarmento-Soares: Contribution to critical revision, adding intellectual content.

Marianna Pinho: Contribution to data collection.

Ronaldo Fernando Martins-Pinheiro: Contribution to data analysis and interpretation.

Sergio Maia Queiroz Lima: Substantial contribution in the concept and design of the study, Contribution to critical revision, adding intellectual content.

Sofia Campiolo: Contribution to data analysis and interpretation.
Priscila Camelier: Substantial contribution in the concept and design of the study; Contribution to manuscript preparation; Contribution to data collection; Contribution to data analysis and interpretation.

\section{Conflicts of Interest}

The authors declares that they have no conflict of interest related to the publication of this manuscript.

\section{References}

ABELL, R., THIEME, M.L., REVENGA, C., BRYER, M., KOTTELAT, M., BOGUTSKAYA, N., COAD, B., MANDRAK, N., BALDERAS, S.L., BUSSING, W., STIASSNY, M.L.J., SKELTON, P., ALLEN, G.R., UNMACK, P., NASEKA, A., NG, R., SINDORF, N., ROBERTSON, J., ARMIJO, E., HIGGINS, J.Y., HEIBEL, T.J., WIKRAMANAYAKE, E., OLSON, D., LÓPEZ, H.L., REIS, R.E., LUNDBERG, J.G., PÉREZ, M.H.S. \& PETRY, P. 2008 Freshwater ecoregions of the world: a new map of biogeographic units for freshwater biodiversity conservation. Bioscience, 58(5):403-414.

ABILHOA, V., BRAGA, R.R., BORNATOWSKI, H., \& VITULE, J.R. 2011. Fishes of the Atlantic Rain Forest streams: ecological patterns and conservation. Changing diversity in changing environment. InTech, Rijeka, p.259-282.

ALBERT, J.S., PETRY, P. \& REIS, R.E. 2011. Major Biogeographic and Phylogenetic Patterns. In: Historical Biogeography of Neotropical Freshwater Fishes (ALBERT J.S. \& R.E. REIS, eds.). Los Angeles: University of California Press, p.21-57.

ALBERT, J.S. \& REIS, R.E. 2011. Introduction to Neotropical Freshwaters. In: Historical Biogeography of Neotropical Freshwater Fishes (ALBERT J.S. \& R.E. REIS, eds.). Los Angeles: University of California Press, p.1-19.

ALLEN, B.S. 2015. Protecting Nature in Federal Systems: States, Private Interests, and Conservation Units in Brazil. Thesis for $\mathrm{PhD}$ in Political Science. University of California, Berkeley, USA.

ALVES, C.B.M. \& LEAL, C.G. 2010. Aspectos da conservação da fauna de peixes da bacia do rio São Francisco em Minas Gerais. MG. Biota, 2(6):26-50.

ALVES, C.B.M., VIEIRA, F., MAGALHÃES, A.L.B. \& BRITO, M.F.G. 2007. Impacts of non-native fish species in Minas Gerais, Brazil: present situation and prospects, in: Ecological and Genetic Implications of Implications of Aquaculture Activities. p.291-314.

ALVES, C.B.M., VIEIRA, F. \& POMPEU, P.S. 2011. A Ictiofauna da Bacia Hidrográfica do Rio São Francisco. In: MMA, Diagnóstico do Macrozoneamento Ecológico-Econômico da Bacia Hidrográfica do Rio São Francisco: Caderno Temático: Biodiversidade. Brasília: SEDR/DZT/MMA. p.226-241.

BARBOSA, J.M. \& SOARES, E.C. 2009. Perfil da ictiofauna da bacia do rio São Francisco: Estudo preliminar. Revista Brasileira de Engenharia de Pesca, 4(1):155-172.

BARbosA, J. M., SOARES, E.C., CINTRA, I.H.A., HERMANN, M. \& ARAÚJO, R.R. 2017. Perfil da ictiofauna da bacia do rio São Francisco. Acta of Fisheries and Aquatic resources, 5(1): 70-90.

BARRETO, S.B., SILVA, A.T., BATALHA-FILHO, H., AFFONSO, P.R.A.M. \& ZANATA, A.M. 2018. Integrative approach reveals a new species of Nematocharax (Teleostei: Characidae). Journal of Fish Biology, 93(6):11511162.

BENINE, R.C., CASTRO, R. \& SANTOS, A.C. 2007. A new Moenkhausia Eigenmann, 1903 (Ostariophysi: Characiformes) from Chapada Diamantina, rio Paraguaçu Basin, Bahia, Northeastern Brazil. Neotropical Ichthyology, $5(3): 259-262$

BERRA, T. M. 2001. Freshwater fish distribution. Academic Press, San Diego, $604 \mathrm{p}$. 
BERTACO, V.A., FERRER, J., CARVALHO, F.R. \& MALABARBA, L.R 2016. Inventory of the freshwater fishes from a densely collected area in South America: a case study of the current knowledge of Neotropical fish diversity. Zootaxa, 4138(3):401-440.

BERTACO, V.A. \& LUCENA, C.A.S. 2006 Two new species of Astyanax (Ostariophysi: Characiformes: Characidae) from eastern Brazil, with a synopsis of the Astyanax scabripinnis species complex. Neotropical Ichthyology, 4(1):53-60.

BICHUETTE, M.E., PINNA, M.C.C. \& TRAJANO, E. 2008. A new species of Glaphyropoma: the first subterranean copionodontine catfish and the first occurrence of opercular odontodes in the subfamily (Siluriformes: Trichomycteridae). Neotropical Ichthyology, 6(3):301-306.

BICHUETTE, M.E. \& RIZZATO, P.P. 2012. A new species of cave catfish from Brazil, Trichomycterus rubbioli sp.n., from Serra do Ramalho karstic area, São Francisco River basin, Bahia State (Silurifomes: Trichomycteridae). Zootaxa, 3480(1):48-66.

BIZERRIL, C.R.S.F. 1994. Análise taxonômica e biogeográfica da ictiofauna de água doce do leste brasileiro. Acta Biológica Leopoldensia, 16(1):51-80.

BOCKMANN, F.A. \& CASTRO, R. 2010. The blind catfish from the caves of Chapada Diamantina, Bahia, Brazil (Siluriformes: Heptapteridae): description, anatomy, phylogenetic relationships, natural history, and biogeography. Neotropical Ichthyology, 8(4):673-706.

BURGER, R.; CARVALHO, F.R. \& ZANATA, A.M. 2019. A new species of Astyanax Baird \& Girard (Characiformes: Characidae) from western Chapada Diamantina, Bahia, Brazil. Zootaxa, 4604(2):369-380.

BURGER, R., ZANATA, A.M. \& CAMELIER, P. 2011. Estudo taxonômico da ictiofauna de água doce da bacia do Recôncavo Sul, Bahia, Brasil. Biota Neotropica, 11(4):273-290.

CAMELIER, P. \& ZANATA, A.M. 2014a. Biogeography of freshwater fishes from the Northeastern Mata Atlântica freshwater ecoregion: distribution, endemism, and area relationships. Neotropical Ichthyology, 12(4):683-698.

CAMELIER, P. \& ZANATA, A.M. 2014b. A new species of Astyanax Baird \& Girard (Characiformes: Characidae) from the Rio Paraguaçu basin, Chapada Diamantina, Bahia, Brazil, with comments on bony hooks on all fins. Journal of Fish Biology, 84(2):475-490.

CASATTI, L., LANGEANI, F. \& CASTRO, R.M.C. 2001. Peixes de riacho do parque estadual Morro do Diabo, bacia do alto rio Paraná, SP. Biota Neotropica, 1(1):1-15.

CASTRO, R.M.C. 1999. Evolução da ictiofauna de riachos sul-americanos: padrões gerais e possíveis processos causais. In: Ecologia de Peixes de Riachos (CARAMASCHI, E.P., Mazzoni, R. \& P.R. Peres-Neto, eds.). Série Oecologia Brasiliensis, vol. VI. PPGE-UFRJ. Rio de Janeiro, Brasil, p.139-155

CETRA, M., SARMENTO-SOARES, L.M \& MARTINS-PINHEIRO, R.F. 2010. Peixes de riachos e novas Unidades de Conservação no sul da Bahia. Pan-American Journal of Aquatic Sciences, 5(1):11-21.

COSTA, W.J.E.M. 2002. Peixes anuais brasileiros: diversidade e conservação. Editora UFPR, Curitiba, 240p.

COSTA, W.J.E.M. 2007. Taxonomic revision of the seasonal South American killifish genus Simpsonichthys (Teleostei: Cyprinodontiformes: Aplocheiloidei: Rivulidae). Zootaxa. 1669(1):1-134.

COSTA, W.J.E.M., AMORIM, P.F. \& BRAGANCA, P.H. 2014 Species limits and phylogenetic relationships of red-finned cryptic species of the seasonal killifish genus Hypsolebias from the Brazilian semi-arid Caatinga. Journal of Zoological Systematics and Evolutionary Research, 52(1):52-58.

COSTA, W.J.E.M. \& LIMA, S.M.Q. 2010. Simpsonichthys ilheusensis a new seasonal killifish of the subgenus Ophthalmolebias from northeastern Brazil. Ichthyological Exploration of Freshwaters, 21(3):205-208

COSTA, W.J.E.M. 2014. Six new species of seasonal killifishes of the genus Cynolebias from the São Francisco river basin, Brazilian Caatinga, with notes on C. porosus. Ichthyological Exploration of Freshwaters, 25(1):79-96.

CRIA (Centro de Referência em Informação Ambiental). 2019. SpeciesLink Network. Fundação de Amparo à Pesquisa do Estado de São Paulo (FAPESP). http://www.splink.org.br (last access in 12/12/2019).
DUDGEON, D., ARTHINGTON, A.H., GESSNER, M.O., KAWABATA, Z.I., KNOWLER, D.J., LÉVÊQUE, C. \& SULLIVAN, C.A. 2006. Freshwater biodiversity: importance, threats, status and conservation challenges. Biological Reviews, 81(2):163-182.

EWERT, M., MENDES, R., RÉDUA, S. \& SEOANE, C.E. 2013. Vozes da permanência: a conservação ambiental alcançada com o sistema da agroflorestal. pp. 393-420. In: STEENBOCK, W., COSTA E SILVA, L., SILVA, R.O., RODRIGUES, A.S., PEREZ-CASSARINO J.\& FONINI, R.(Orgs.). Agrofloresta, ecologia e sociedade. Curitiba, Kairós.

FERREIRA, F.S., VICENTIN, W., COSTA, F.E.D.S. \& SÚAREZ, Y.R. 2014. Trophic ecology of two piranha species, Pygocentrus nattereri and Serrasalmus marginatus (Characiformes, Characidae), in the floodplain of the Negro River, Pantanal. Acta Limnologica Brasiliensia, 26(4):381-391.

FRICKE, R., ESCHMEYER, W.N. \& VAN DER LAAN, R. (eds.) 2020. Eschmeyer's catalog of fishes: genera, species, references. http:// researcharquive.calacademy.org/research/ichthyology/catalog/fishcatmain. asp. (last access in 30/12/2020).

GARAVELLO, J C. 1977. Systematics and geographical distribution of the genus Parotocinclus Eigenmann \& Eigenmann, 1889 (OSTARIOPHYSI, LORICARIIDAE). Arquivos de Zoologia, 28:1-37.

GODINHO, A.L. \& GODINHO, H.P., 2003. Breve visão do São Francisco. In: Águas, peixes e pescadores do São Francisco das Minas Gerais (SATO, Y., FENERICH-VERANI, N., GODINHO, H.P. \& A.L. GODINHO, eds). Belo Horizonte: PUC Minas, p.15-23.

GODINHO, A.L. \& KYNARD, B., 2006. Migration and Spawning of Radiotagged Zulega Prochilodus argenteus in a Dammed Brazilian River. Transactions of the American Fisheries Society, 135:811-824.

GONÇALVES, C.S \& BRAGA, F.M.S. 2012. Changes in ichthyofauna composition along a gradient from clearwaters to blackwaters in coastal streams of Atlantic forest (southeastern Brazil) in relation to environmental variables. Neotropical Ichthyology, 10(3):675-684.

HALES, J. \& PETRY, P. 2013. Freshwater Ecoregions of the world: Northeastern Mata Atlantica ecoregion. Available from: http://www.feow.org/ecoregions/ details/northeastern_mata_atlantica (last access in 10/07/2018).

HORTAL, J., DE BELLO, F., DINIZ-FILHO, J.A., LEWINSOHN, T.M., LOBO, J.M. \& LADLE, R.J. 2015. Seven Shortfalls that Beset Large-Scale Knowledge of Biodiversity. Annual Review of Ecology, Evolution, and Systematics. Syst., 46:523-549.

IBGE, $2019 \mathrm{https} / / /$ cidades.ibge.gov.br/brasil/ba/panorama (last access in $17 / 08 / 2020)$

ICMBIO, 2014. Instituto Chico Mendes. List of Endangered Species. https:// www.icmbio.gov.br/portal/faunabrasileira/avaliacao-do-risco-deextincao?start $=30$ (last access in 17/08/2020).

ICMBIO, 2018. Livro Vermelho da Fauna Brasileira Ameaçada de Extinção: Volume VI -Peixes. 1.ed., Brasília, DF: ICMBio/MMA.

IUCN, 2012. IUCN Red List Categories and Criteria version 3.1. http:// www.iucnredlist.org/technical-documents/categories-and-criteria/2001categories-criteria (last access in 11/07/2018).

KOHLER, H.C. 2003. Aspectos geoecológicos da bacia hidrográfica do São Francisco (primeira aproximação na escala 1: 1000 000). In: Águas, peixes e pescadores do São Francisco das Minas Gerais. Belo Horizonte: Editora PUC Minas, p.25-35.

LANGEANI, F.L., BUCKUP, P.A., MALABARBA, L.R., PY-DANIEL, L.H.R, LUCENA, C.A., ROSA, R.S., ZUANON, J.A.S., LUCENA, Z.M.S., BRITTO, M.R., OYAKAWA, O.T. \& GOMES-FILHO, G. 2009. Peixes de água Doce. In: Estado da arte e perspectivas para a Zoologia no Brasil. (Rocha, R.M. \& W.A.P. Boeger, orgs.). Universidade Federal do Paraná, Curitiba, Brasil, p.211-230

LÉVÊQUE, C., OBERDORFF, T., PAUGY, D., STIASSNY, M. \& TEDESCO, P.A. 2008. Global diversity of fish (Pisces) in freshwater. Hydrobiologia, 595:545-567.

LIMA, F.C. \& BRITSKI, H.A. 2007. Salminus franciscanus, a new species from the rio São Francisco basin, Brazil (Ostariophysi: Characiformes: Characidae). Neotropical Ichthyology, 5(3):237-244. 
LIMA, S.M.Q., RAMOS, T.P.A., DA SILVA, M.J. \& DE SOUZA ROSA, R. 2017. Diversity, distribution, and conservation of the Caatinga fishes: advances and challenges. In Caatinga (pp. 97-131). Springer, Cham.

LUCENA, Z.M.S. \& LUCENA, C.A.S. 2019. A new glass tetra species of Phenacogaster from the rio Salitre, rio São Francisco drainage, Brazil (Characiformes: Characidae). Neotropical Ichthyology, 17(1):e180134.

MALABARBA, L.R., LIMA, F.C., \& WEITZMAN, S.H. 2004. A new species of Kolpotocheirodon (Teleostei: Characidae: Cheirodontinae: Compsurini) from Bahia, northeastern Brazil, with a new diagnosis of the genus. Proceedings of the Biological Society of Washington, 117(3):317-329.

MATTOS, J.L. \& COSTA, W.J.E.M. 2018. Three new species of the 'Geophagus' brasiliensis species group from the northeast Brazil (Cichlidae, Geophagini). Zoosystematics and Evolution, 94(2):325-337.

MATTOS, J.L., COSTA, W.J.E.M. \& SANTOS, A.C. 2015. Geophagus diamantinensis, a new species of the G. brasiliensis species group from Chapada Diamantina, north-eastern Brazil (Cichlidae: Geophagini). Ichthyological Exploration of Freshwaters, 26(3):209-220.

MENEZES, N.A. 1996a. Methods for assessing freshwater fish diversity. In Biodiversity in Brazil (BICUDO, C.E.M. \& N.A. MENEZES, eds.). CNPq, São Paulo, p.289-295.

MENEZES, N.A. 1996b. Padrões de distribuição da biodiversidade da Mata Atlântica do sul e sudeste brasileiro: peixes de água doce. In Workshop Padrões de Biodiversidade da Mata Atlântica do Sudeste e Sul do Brasil, Campinas.

MIRANDE, J.M. 2010. Phylogeny of the family Characidae (Teleostei: Characiformes): from characters to taxonomy. Neotropical Ichthyology, 8(3):385-568.

MMA - Ministério do Meio Ambiente. 2004. Lista nacional das espécies de invertebrados aquáticos e peixes ameaçados de extinção com categorias da IUCN. Instrução Normativa no. 5 de 21 de maio de 2004. Diário Oficial da União 102:102-142.

MMA - Ministério do Meio Ambiente. 2014. Portaria $N^{\circ} 445$, de 17 de dezembro de 2014. Lista Nacional Oficial de Espécies da Fauna Ameaçadas de Extinção Peixes e Invertebrados Aquáticos. Anexo I, Anexo II.

MYERS, G.S. 1949. Salt-tolerance of fresh-water fish groups in relation to zoogeographical problems. Bijdragen tot de Dierkunde, 28(1949):315-322.

NOGUEIRA, C., BUCKUP, P.A., MENEZES, N.A., OYAKAWA, O.T., KASECKER, T.P., RAMOS-NETO, M.B., SILVA, J.M.C. 2010. Restrictedrange fishes and the conservation of Brazilian freshwaters. PLoS ONE, $5(6): 1-10$

OLIVEIRA, D.C.D. \& BENNEMANN, S.T., 2005. Ictiofauna, recursos alimentares e relações com as interferências antrópicas em um riacho urbano no sul do Brasil. Biota Neotropica, 5(1):95-107.

OLIVEIRA, L.M.A.; ZANATA, A.M.; TENCATT, L.F.C. \& BRITTO, M.R. 2013. A new species of Aspidoras (Siluriformes: Callichthyidae) from a small coastal drainage in northeastern Brazil. Neotropical Ichthyology, 15(1):e160118.

PAIVA, M.P. 1982. Grandes represas do Brasil. Brasilia; EDITERRA, 304p.

PEIXOTO, L.A.W. \& WOSIACKI, W.B. 2016. Eigenmannia besouro, a new species of the Eigenmannia trilineata species-group (Gymnotiformes: Sternopygidae) from the rio São Francisco basin, northeastern Brazil. Zootaxa, 4126(2):262-270.

POLAZ, C.N.M. 2014. Brazilian Action Plans for freshwater fishes. Saving freshwater fishes and habitats. Newsletter of the IUCN SSC/WI Freshwater Fish Specialist Group, 4:15.

POMPEU, P.D.S. \& GODINHO, H.P. 2006. Effects of extended absence of flooding on the fish assemblages of three floodplain lagoons in the middle São Francisco River, Brazil. Neotropical Ichthyology, 4(4):427-433.

RAMOS, T.P.A., RAMOS, R.T.C., \& RAMOS, S.A.Q.A. 2014. Ichthyofauna of the Parnaíba river Basin, Northeastern Brazil. Biota Neotropica, 14(1):1-8.

REIS, R.E. 2013. Conserving the freshwater fishes of South America. International Zoo Yearbook 47(1):65-70.

REIS, R.E., ALBERT, J.S., DI DARIO, F., MINCARONE, M.M., PETRY, P. \& ROCHA, L.A. 2016. Fish biodiversity and conservation in South America. Journal of Fish Biology 89(1):12-47.
RIBEIRO, A.C., 2006. Tectonic history and the biogeography of the freshwater fishes from the coastal drainages of eastern Brazil: an example of faunal evolution associated with a divergent continental margin. Neotropical Ichthyology, 4(2):225-246.

RIBEIRO, F.R.V. \& LUCENA, C.A.S.D. 2006. A new species of Pimelodus LaCépède, 1803 (Siluriformes: Pimelodidae) from the rio São Francisco drainage, Brazil. Neotropical Ichthyology, 4(4):411-418.

ROSA, R.S., MENEZES, N.A., BRITSKI, H.A., COSTA, W.J.E.M. \& GROTH, F. 2003. Diversidade, padrões de distribuição e conservação dos peixes da Caatinga. In: Ecologia e conservação da Caatinga (Leal, I.L., Tabareli, M., J.M.C. da Silva, eds.). Recife: Editora Universitária da UFPE, Brasil, p.135-180.

SALA, O.E., CHAPIN, F.S., ARMESTO, J.J., BERLOW, E., BLOOMFIELD, J., DIRZO, R., HUBER-SANWALD, E., HUENNEKE, L.F., JACKSON, R.B., KINZIG, A. \& LEEMANS, R., 2000. Global biodiversity scenarios for the year 2100. Science, 287(5459):1770-1774.

SANTOS, A.C.A. 2005. Peixes. In: JUNCÁ, F.A., FUNCH, L. \& ROCHA, W. (Eds.). Biodiversidade e conservação da Chapada Diamantina. Brasília: Ministério do Meio Ambiente. 411p.

SARMENTO-SOARES, L.M., R. MAZZONI \& MARTINS-PINHEIRO, R. F. 2007. A fauna de peixes na bacia do Rio Peruípe, extremo Sul da Bahia. Biota Neotropica, 7(3):291-308.

SARMENTO-SOARES, L.M., R. MAZZONI \& MARTINS-PINHEIRO, R. F. 2009a. A fauna de peixes nas bacias litorâneas da Costa do Descobrimento, Extremo Sul da Bahia, Brasil. Sitientibus Série Ciências Biológicas 9(2/3):139-1575.

SARMENTO-SOARES, L.M., LEHMANN P.A. \& MARTINS-PINHEIRO, R. F. 2009b. Parotocinclus arandai, a new species of hypoptopomatine catfish (Siluriformes: Loricariidae) from the upper rios Jucuruçu and Buranhém,States of Bahia and Minas Gerais, Brazil. Neotropical Ichthyology, 7(2):191-198.

SARMENTO-SOARES, L.M. \& MARTINS-PINHEIRO, R.F. 2013. Glanidium botocudo, a new species from the rio Doce and rio Mucuri, Minas Gerais, Brazil (Siluriformes: Auchenipteridae) with comments on taxonomic position of Glanidium bockmanni. Neotropical Ichthyology, 11(2):265-274.

SARMENTO-SOARES, L.M. \& MARTINS-PINHEIRO, R.F. 2017. Unidades de Conservação e a água: a situação das áreas protegidas de Mata Atlântica ao norte do Espírito Santo - Sudeste do Brasil. BioBrasil, ICMBio.

SARMENTO-SOARES, L.M., ZANATA, A.M. \& MARTINS-PINHEIRO, R.F. 2011. Trichomycterus payaya, new catfish (Siluriformes: Trichomycteridae) from headwaters of rio Itapicuru, Bahia, Brazil. Neotropical Ichthyology, 9(2):261-271.

SATO, Y. \& GODINHO, H.P., 1999. Peixes da bacia do rio São Francisco. Estudos ecológicos de comunidades de peixes tropicais. São Paulo: Edusp, p.401-413.

SATO, Y. \& GODINHO, H.P. 2003. Migratory fishes of the São Francisco River. In: Migratory Fishes of South America: Biology, Fisheries and Conservation Status (Carolsfeld, J., Harvey, B., Ross, C. \& Baer A., eds). IDRC, Victoria, 380p.

SEMA. 2017. Portaria n ${ }^{\circ} 37$ de 15 de agosto de 2017. Lista Oficial das Espécies da Fauna Ameaçadas de Extinção do Estado da Bahia. Secretaria do Meio Ambiente de Bahia - SEMA.

SIBIP/NEODAT III. 2019. Sistema Brasileiro de Informações sobre Biodiversidade de Peixes. Sistema Nacional de Informações sobre Coleções Ictiológicas. http://www.mnrj.ufrj.br/pronex/ (last access in 10/12/2019).

SILVEIRA, L.F., BEISIEGEL, B.D.M., CURCIO, F.F., VALDUJO, P.H., DIXO, M., VERDADE, V.K., MATTOX, G.M.T. \& CUNNINGHAM, P.T.M. 2010. Para que servem os inventários de fauna? Estudos avançados, 24(68):173-207.

TEDESCO, P.A., BEAUCHARD, O., BIGORNE, R., BLANCHET, S., BUISSON, L., CONTI, L., CORNU, J.F., DIAS, M.S., GRENOUILLET, G., HUGUENY, B., JÉZÉQUEL, C., LEPRIEUR, F., BROSSE, S. \& JÉZÉQUEL, C. 2017. A global database on freshwater fish species occurrence in drainage basins. Scientific data, 4:170141. 
TRAJANO, E. \& BICHUETTE, M.E. 2010. Relevância de cavernas: porque estudos ambientais espeleobiológicos não funcionam? Espeleo-Tema (São Paulo), 21:105-112.

WEITZMAN, S.H. \& VARI, R.P., 1988. Miniaturization in South American freshwater fishes; an overview and discussion. Proc. Biol. Soc. Wash., 101:444-465.

VARI, R.P., ZANATA, A.M. \& CAMELIER, P. 2010. New species of Cyphocharax (OSTARIOPHYSI: CHARACIFORMES: CURIMATIDAE) from the rio de Contas drainage, Bahia, Brazil. Copeia, 3:382-387.

ZANATA, A.M.; BURGER, R. \& CAMELIER, P. 2018. Two new species of Astyanax Baird \& Girard (Characiformes: Characidae) from the upper rio Paraguaçu basin, Chapada Diamantina, Bahia, Brazil. Zootaxa, 4438(3):471-490.

ZANATA, A.M. \& CAMELIER, P. 2008. Two new species of Astyanax (Characiformes: Characidae) from upper rio Paraguaçu and rio Itapicuru basins, Chapada Diamantina, Bahia, Brazil. Zootaxa, 1908(1):28-40.

ZANATA, A.M. \& CAMELIER, P. 2009. Astyanax vermilion and Astyanax burgerai: new characid fishes (Ostariophysi: Characiformes) from Northeastern Bahia, Brazil. Neotropical Ichthyology, 7(2):175-184.

ZANATA, A.M. \& CAMELIER, P. 2010. Hyphessobrycon brumado: a new characid fish (OSTARIOPHYSI: CHARACIFORMES) from the upper rio de Contas drainage, Chapada Diamantina, Bahia, Brazil. Neotropical Ichthyology, 8(4):771-777.

ZANATA, A.M. \& CAMELIER, P. 2014. A new species of Characidium (Characiformes: Crenuchidae) from small coastal drainages in northeastern Brazil, with remarks on the pseudotympanum of some species of the genus. Neotropical Ichthyology, 12(2):333-342.
ZANATA, A.M. \& CAMELIER, P. 2015. Two new species of Characidium Reinhardt (Characiformes: Crenuchidae) from northeastern Brazilian coastal drainages. Neotropical Ichthyology, 13(3):487-498.

ZANATA, A.M., LIMA, F.C.T., DI DARIO, F. \& GERHARD, P. 2017. A new remarkable and Critically Endangered species of Astyanax Baird \& Girard (Characiformes: Characidae) from Chapada Diamantina, Bahia, Brazil, with a discussion on durophagy in the Characiformes. Zootaxa, 4232(4):491-510.

ZANATA, A.M. \& PITANGA, B.R. 2016. A new species of Hypostomus Lacépède, 1803 (Siluriformes: Loricariidae) from rio Itapicuru basin, Bahia State, Brazil. Zootaxa, 4137(2):223-232.

ZANATA, A.M., SARMENTO-SOARES, L. M. \& MARTINS-PINHEIRO, R. F. 2015. A new species of Characidium Reinhardt (Ostariophysi: Characiformes: Crenuchidae) from coastal rivers in the extreme south of Bahia, Brazil. Zootaxa 4040(3):371-383.

ZANATA, A.M. \& SERRA, J.P. 2010. Hasemania piatan, a new characid species (CHARACIFORMES: CHARACIDAE) from headwaters of rio de Contas, Bahia, Brazil. Neotropical Ichthyology, 8(1):21-26.

ZAWADZKI, C.H., OYAKAWA, O.T. \& BRITSKI, H.A. 2017. Hypostomus velhochico, a new keeled Hypostomus Lacépède, 1803 (Siluriformes: Loricariidae) from the rio São Francisco basin in Brazil. Zootaxa, 4344(3):560-572.

Received: 04/02/2020

Revised: 18/08/2020

Accepted: 18/08/2020

Published online: 21/09/2020 